\title{
Les Jeux olympiques de Berlin dans l'arène médiatique montréalaise
}

Distance, censure et prise de parole

Xavier Boileau, Jeanne Boucher Lauzon, Micheline Cambron, Dominique Marquis, Alex Giroux, Eve Léger-Bélanger, Jérémi Perrault, Yosef

Robinson and Marilou St-Pierre

\section{OpenEdition}

\section{Journals}

\section{Electronic version}

URL: http://journals.openedition.org/belphegor/909

DOI: 10.4000/belphegor.909

ISSN: 1499-7185

Publisher

LPCM

\section{Electronic reference}

Xavier Boileau, Jeanne Boucher Lauzon, Micheline Cambron, Dominique Marquis, Alex Giroux, Eve Léger-Bélanger, Jérémi Perrault, Yosef Robinson and Marilou St-Pierre, « Les Jeux olympiques de Berlin dans l'arène médiatique montréalaise », Belphégor [Online], 15-1 | 2017, Online since 06 July 2017, connection on 01 May 2019. URL : http://journals.openedition.org/belphegor/909 ; DOI : 10.4000/belphegor.909

This text was automatically generated on 1 May 2019.

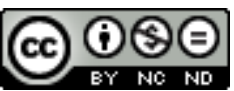

Belphégor est mis à disposition selon les termes de la Licence Creative Commons Attribution - Pas d'Utilisation Commerciale - Pas de Modification 4.0 International. 


\title{
Les Jeux olympiques de Berlin dans l'arène médiatique montréalaise
}

\author{
Distance, censure et prise de parole \\ Xavier Boileau, Jeanne Boucher Lauzon, Micheline Cambron, Dominique \\ Marquis, Alex Giroux, Eve Léger-Bélanger, Jérémi Perrault, Yosef \\ Robinson and Marilou St-Pierre
}

1 Notre étude s'inscrit dans le cadre plus large de travaux sur la presse montréalaise de l'entre-deux-guerres ${ }^{1}$. À ce titre, notre analyse de la couverture des Jeux olympiques de Berlin dans les principaux journaux montréalais vise à la fois à éclairer le retentissement des JO dans la société québécoise de 1936, à cerner le fonctionnement du système des journaux montréalais durant les deux semaines des Jeux ${ }^{2}$ et, en croisant nos résultats avec ceux de chercheurs français, belges, suisses et québécois, à développer les études comparées sur la presse francophone. On verra que ces trois objectifs se révèlent complémentaires. Autour de la couverture des Jeux olympiques de Berlin, événement sportif international dont la portée est sociale, politique et éthique, s'entrecroisent en effet des discours, des motifs narratifs et une certaine façon de faire le journal qui débordent la dimension événementielle de l'objet traité, ce qui permet de dégager la dynamique médiatique montréalaise, lisible dans la différenciation des pratiques, les tensions polémiques et aussi les silences des uns et des autres.

2 Notre dépouillement sur les Jo faisait suite à une analyse systématique des journaux visant à connaître leurs factures respectives, leurs orientations commerciales et culturelles et le lectorat visé tel que ces dernières le donnent à voir ${ }^{3}$. Nous avons aussi pu compter sur un travail préalable, réalisé à partir de dépouillements longitudinaux portant sur la présence de l'Allemagne dans La Presse et La Patrie autour de quatre événements : l'élection d'Hitler, les Jeux olympiques de Berlin, l'Anschluss et l'invasion de la France ${ }^{4}$. Nous disposions donc de plusieurs informations et, postulant que la presse est à la fois signe et agent des changements culturels à l'œuvre dans la collectivité, nous avons rapidement formulé l'hypothèse que les JO constituaient un révélateur autour duquel se cristallisaient les clivages identitaires, associés aux divers discours nationalistes en circulation (canadien-français, impérialiste britannique, pro-canadien, sioniste, 
fasciste italien), et les clivages politiques, entés sur des positions idéologiques ou partisanes.

3 Par ailleurs, une première rencontre internationale, réalisée aux deux tiers $d u$ dépouillement, nous a fait découvrir le caractère très contrasté des couvertures québécoise et européenne. Quelle déception! Nous n'avions pas au Québec de grands reportages illustrés, de textes signés de noms prestigieux, d'investissement littéraire substantiel dans des narrations vivantes! L'absence de signatures fortes et de textes flamboyants, la rareté des photographies d'action et le caractère convenu de l'iconographie, la relative pauvreté quantitative et qualitative de la couverture, en apparence réduite à des tableaux sportifs, nous ont incités à chercher à comprendre les sources de ces particularités en nous reportant aux résultats quantitatifs engrangés. Pourquoi l'événement prévisible que constituaient les JO a-t-il connu une couverture aussi pauvre? Y a-t-il des éléments qui se démarquent au sein de cette grisaille? Comment peut-on les repérer? Quel sens peut-on leur donner? Quel instantané du système des journaux cette couverture nous offre-elle?

4 Après avoir décrit notre corpus et situé les Jeux olympiques dans le contexte québécois de 1936, nous présentons, dans une première section, la couverture des JO en la situant sur fond des pratiques habituelles de chacun des journaux placés au centre de l'étude à l'aide d'une description statistique. La seconde section vise à caractériser les discours en s'attachant à leurs modalités énonciatives. Qui parle? À qui ? Sur quel ton? Dans quel contexte énonciatif ou pragmatique ? Nous abordons ainsi la question des sources et celle du traitement des dépêches des agences de presse, celle des signatures, et analysons enfin brièvement diverses formes d'expression du jugement à propos des JO. Nous proposons en fin de course quelques conclusions liées à la nature du traitement des Jeux, que notre analyse a révélée non réductible aux lignes de fractures usuelles du système des journaux - type de publication, langue et revendication d'une identité, rattachement partisan. Celles-ci soulèvent des interrogations quant aux clés d'interprétation des Jeux offertes ou non par les journaux, dans les chroniques, les éditoriaux, les lettres et les correspondances écrites depuis l'Europe qui racontent autrement les Jeux, dévoilant une instrumentalisation politique fort éloignée des principes humanistes explicitement convoqués dans la mystique olympique.

\section{Particularités du contexte national québécois : sport et élections}

5 Une brève esquisse du contexte québécois s'impose avant de présenter les résultats de l'analyse. Les deux semaines de la tenue des JO coïncident au Québec avec une campagne électorale provinciale, qui met aux prises la nouvelle Union nationale, parti de coalition très hétérogène qui regroupe des conservateurs nationalistes et des libéraux au sens classique, que nous situerions au centre-gauche aujourd'hui, dirigé par Maurice Duplessis, face au Parti libéral, usé par le pouvoir et miné par une corruption éhontée. Il importe de dire que les idéaux démocratiques sont alors portés par ce même Parti libéral (éducation, sécurité sociale, vote des femmes) et que les grands ténors nationalistes sont plutôt favorables à un corporatisme propre à rétablir l'ordre en des temps difficiles, et penchent pour l'Union nationale. La crise économique ne s'est pas encore tout à fait résorbée et plusieurs groupes marginaux sont présents sur la place publique, dont le Parti ouvrier progressiste, communiste, et le Parti national social chrétien, fasciste ${ }^{5}$. La campagne 
occupe une place importante dans les journaux, ce qui fait dire à Elmer W. Ferguson du Montreal Herald que les lecteurs ne bénéficient pas d'informations suffisantes sur les JO à cause de ce déroulement simultané ${ }^{6}$. Par ailleurs, les unes des journaux, en particulier de ceux de grande diffusion comme La Patrie, La Presse et le Star, sont largement occupées par la Guerre d'Espagne et les tractations diplomatiques auxquelles celle-ci donne lieu, témoignant de la crainte d'un second conflit mondial, et ne laissent pas, dans l'ensemble du corpus, de place aux Jeux, sauf en trois occasions.

Les relations «sportives » entre le Québec et les JO méritent aussi d'être précisées. Le Canada participe aux JO modernes depuis ses débuts, quoiqu'il ait été représenté au Comité International Olympique (CIO) par la Grande-Bretagne jusqu'en $1907^{7}$. Même si le pays obtient le plein exercice de sa souveraineté en matière de politique étrangère en 1931, le Comité olympique canadien adopte sans discussion la position de la GrandeBretagne à l'endroit d'un boycott éventuel des Jeux de Berlin en 1936. Certains formulent des critiques à l'endroit du Comité pour son processus de sélection des athlètes de l'équipe canadienne et leur financement en 1932 et $1936^{\circ}$. Ce n'est qu'après les JO de Berlin que le gouvernement canadien s'engagera à faire respecter une certaine éthique dans la promotion du sport olympique - la chute dramatique du nombre de médaillés canadiens de 1932 à 1936 semble avoir constitué un facteur clé dans cette décision.

7 Au Québec, par ailleurs, la grande majorité des clubs d'athlétisme sont anglophones jusqu'au milieu des années $1960^{\circ}$, moment où l'athlétisme gagne de la popularité dans les milieux francophones et où les noms des associations se francisent ${ }^{10}$. Grâce au Montreal Amateur Athletic Association (MAAA), actif dès 1881, les anglophones québécois sont quant à eux très présents dans les délégations olympiques canadiennes dès le début du XXe siècle ${ }^{11}$ : au moins un athlète québécois participe à chacun des Jeux tenus entre 1904 et 1936, à l'exception de ceux de 1920. Le seul athlète francophone à se démarquer est Étienne Desmarteau, policier à Montréal, qui gagne l'or au lancer du poids en 1904. La proximité du milieu anglophone avec les disciplines athlétiques se reflète dans les journaux: la Gazette et le Star présentent les résultats sportifs du M.A.A.A. de façon sporadique, alors que les journaux francophones ne couvrent pas l'athlétisme amateur ${ }^{12}$.

\section{Corpus et méthodologie}

8 Notre corpus était constitué de journaux et de revues écrits en français et en anglais, mais aussi en yiddish et en italien ${ }^{13}$. Ceux-ci appartiennent à différentes mouvances politiques - les journaux libéraux sont au centre ou au centre gauche et s'opposent à la presse conservatrice ; les journaux nationalistes sont plutôt conservateurs, mais pas toujours. Le lectorat varie en outre considérablement d'une publication à l'autre, à la fois en termes de tirage ${ }^{14}$ et de distribution géographique ${ }^{15}$. La période analysée s'étend du $1^{\mathrm{er}}$ août, date officielle de l'ouverture des Jeux, jusqu'au 17 août 1936, lendemain de leur clôture. Nous avons opéré deux types de dépouillement: le premier se veut une analyse exhaustive et touche toute la matière du journal ; le second est longitudinal et concerne exclusivement les JO de Berlin et tout ce qui s'y rattache. Nous avons étudié exhaustivement cinq quotidiens: The Montreal Daily Star ${ }^{16}$, La Patrie ${ }^{17}$, Le Canada ${ }^{18}$, L'Illustration Nouvelle ${ }^{19}$ et Der Keneder Odler $^{20}$ ainsi que le magazine mensuel La Revue moderne ${ }^{21}$. Il s'agissait de relever et d'analyser tous les éléments ${ }^{22}$ du journal en considérant comme "élément » toute information autonome, clairement circonscrite, qu'il s'agisse d'une nouvelle, d'une rubrique ou encore d'une photo-vignette ${ }^{23}$, d'une caricature ou d'une publicité. À partir 
des traits saillants identifiés dans les dépouillements systématiques, nous avons tiré profit de l'étude longitudinale menée antérieurement sur les JO dans La Presse ${ }^{24}$ et l'avons élargie à six autres journaux, L'Autorité $e^{25}$, Le Devoir ${ }^{26}$, The Gazette ${ }^{27}$, The Montreal Herald ${ }^{28}$ Le Petit journal ${ }^{29}$ et $L^{\prime} I t a l i a^{30}$. Notre étude repose donc sur l'analyse des journaux montréalais les plus importants, toutes orientations politiques ou partisanes confondues. On remarquera qu'il n'existe pas à Montréal, ni au Québec, de périodique exclusivement consacré au sport en 1936. Il importe donc de lire les résultats des analyses qui suivent en tenant compte du caractère généraliste des publications étudiées.

9 Les dépouillements ont été réalisés en utilisant une banque de données via une plateforme web. Les variables relatives au contenu et aux médias que nous avons utilisées pour le portrait quantitatif se rapprochent de celles suggérées par Jean de Bonville ${ }^{31}$. Ont été relevés pour chaque élément dépouillé : la nature de l'élément (nouvelle, chronique, éditorial, etc.), le type de section où il se trouvait (sports, finances, etc.), son titre, l'emplacement et la surface occupée sur la page, et enfin, la source (agence de presse, ville ou signature selon les cas). Les codeurs identifiaient, pour chaque élément, l'objet ou les objets traités parmi une liste pré-établie (cinéma, classe ouvrière, fait divers, guerre/militaire, mode, musique, politique, radio, etc.), au sein de laquelle se trouvaient les JO, désignés comme événement nodal. Les éléments de cette dernière catégorie ont également été analysés selon d'autres variables liées à la vie culturelle (essentiellement la mention de personnalités canadiennes et étrangères) et à la langue (mode discursif, figures de style, usages pronominaux, temps et modes verbaux). Nous avons également relevé les phrases ou les expressions marquantes comme des matériaux bruts, sous forme de citation donc, dans une section intitulée "Résumé isomorphe », laquelle s'est révélée particulièrement féconde. Nous nous sommes également appuyés sur les rapports synthétiques préparés par les codeurs et les codeuses qui ont dépouillé les différentes publications, afin de nuancer et d'enrichir ces résultats quantitatifs.

\section{La couverture des Jeux en chiffres}

10 Notre portrait général de la couverture des Jeux olympiques de Berlin s'appuie sur l'analyse de quatre des cinq quotidiens ayant fait l'objet d'un dépouillement exhaustif : le Star, La Patrie, Le Canada et L'Illustration nouvelle. En effet, l'absence de couverture sportive dans le Keneder Odler et le peu d'éléments portant sur les JO qui s'y trouvent nous ont incités à réserver à ce journal un traitement exclusivement qualitatif. Par ailleurs, il importe de souligner que la surface de la section sport n'est pas modifiée durant les Jeux, c'est-à-dire que la couverture des JO qui se fait dans les pages sportives n'a pas entraîné une augmentation de la surface consacrée aux sports. Cet état de fait a fortement pesé sur notre traitement statistique, d'autant que $93,2 \%$ de tout ce qui est relatif aux JO se retrouve dans la section des sports. 
Tableau 1. Répartition de la couverture des Jeux olympiques de Berlin : nombre d'éléments selon la date et le journal, du $1^{\text {er }}$ au 17 août 1936

\begin{tabular}{|c|c|c|c|c|}
\hline & $\begin{array}{l}\text { Montreal Daily } \\
\text { Star }\end{array}$ & La Patrie: & Le Canada & $\begin{array}{c}\text { Lillustration } \\
\text { nouvelle: }\end{array}$ \\
\hline Samedi, 1er août & 10 & 2 & 3 & 2 \\
\hline Dimanche, 2 août & NSP & 10 & NSP & NSP \\
\hline Lundi, 3 août & 21 & 5 & 5 & 4 \\
\hline Mardi, 4 août & 16 & 12 & 6 & 7 \\
\hline Mercredi, 5 août & 19 & 15 & 6 & 6 \\
\hline Jeudi, 6 août & 21 & 12 & 8 & 6 \\
\hline Vendredi, 7 aout & 19 & 11 & 6 & 9 \\
\hline Samedi, 8 août & 16 & 2 & 10 & 8 \\
\hline Dimanche, 9 aoút & NSP & 6 & NSP & NSP \\
\hline Lundi, 10 août & 20 & 10 & 7 & 5 \\
\hline Mardi, 11 août & 19 & 12 & 8 & 8 \\
\hline Mercredi, 12 aout & 17 & 6 & 5 & 4 \\
\hline leudi, 13 août & 16 & 8 & 3 & 6 \\
\hline Vendredi, 14 août & 15 & 8 & 4 & 6 \\
\hline Samedi, 15 août & 15 & 2 & 3 & 1 \\
\hline Dimanche, 16 août & NSP & 3 & NSP & NSP \\
\hline Lundi, 17 août & 8 & 1 & 4 & 4 \\
\hline Totaux & 232 & 125 & 78 & 76 \\
\hline
\end{tabular}

11 En examinant le tableau 1, nous constatons qu'au cours des deux semaines qu'ont duré les compétitions olympiques, soit du $1^{\mathrm{er}}$ au 17 août 1936, la couverture offerte par les journaux a été relativement stable, exception faite de La Patrie. En effet, pour ce quotidien, la couverture des Jeux a été passablement moins importante durant la deuxième semaine d'activités, en plus de se faire discrète dans les parutions du samedi et dimanche, pourtant les plus volumineuses.

Si nous nous tournons du côté de l'espace occupé sur la page par les éléments portant sur les Jeux olympiques (voir graphique 1), nous remarquons que le Star et Le Canada se démarquent par des éléments relativement courts, couvrant de 2 à $5 \%$ de la page, comparativement à L'Illustration et à La Patrie qui comportent une plus grande proportion d'articles couvrant de 5 à $15 \%$ d'une page. Toutefois, en comparant la couverture des Jeux avec le reste du contenu rédactionnel des journaux, nous remarquons que pour l'ensemble des journaux étudiés, mais particulièrement pour Le Star et La Patrie, la couverture des Jeux est constituée d'éléments qui occupent en moyenne une plus grande surface de la page que les autres éléments du journal. Parmi les éléments du Star sur les JO, $36,8 \%$ couvrent entre 5 et $15 \%$ de la page, tandis que pour le reste de la matière publiée la proportion des textes situés dans cette fourchette est de seulement 19,7\%. 


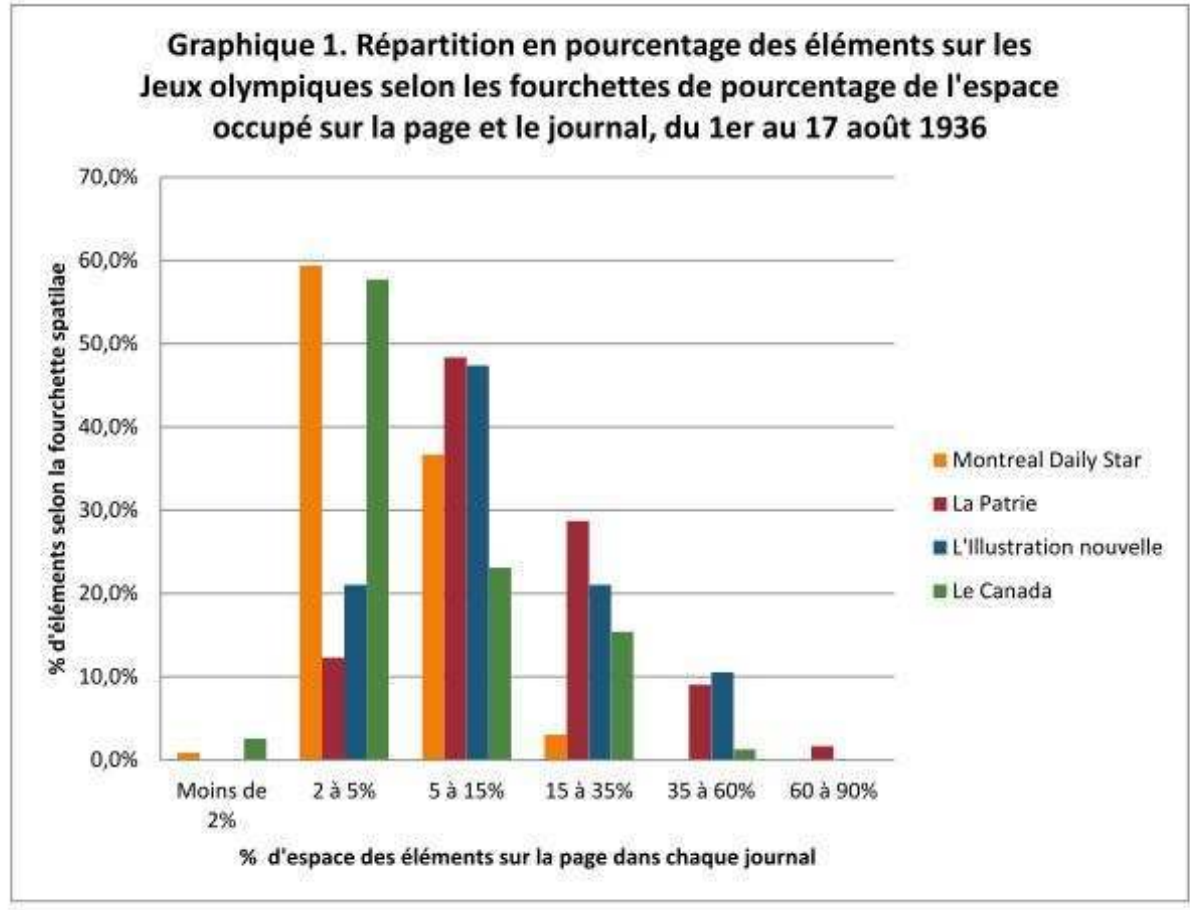

Comme l'illustre le Graphique 2, les éléments portant sur les JO occupent, dans La Patrie, une surface proportionnellement plus grande que les autres éléments. Ainsi, 28,7\% des éléments occupent entre 15 et $35 \%$ de la page alors que seulement $17 \%$ des éléments portant sur d'autres objets le font. Malgré ce que donne à voir la faible proportion d'éléments sur les JO dans les journaux (Tableau 1), ceux-ci peuvent néanmoins infléchir la facture générale des numéros.

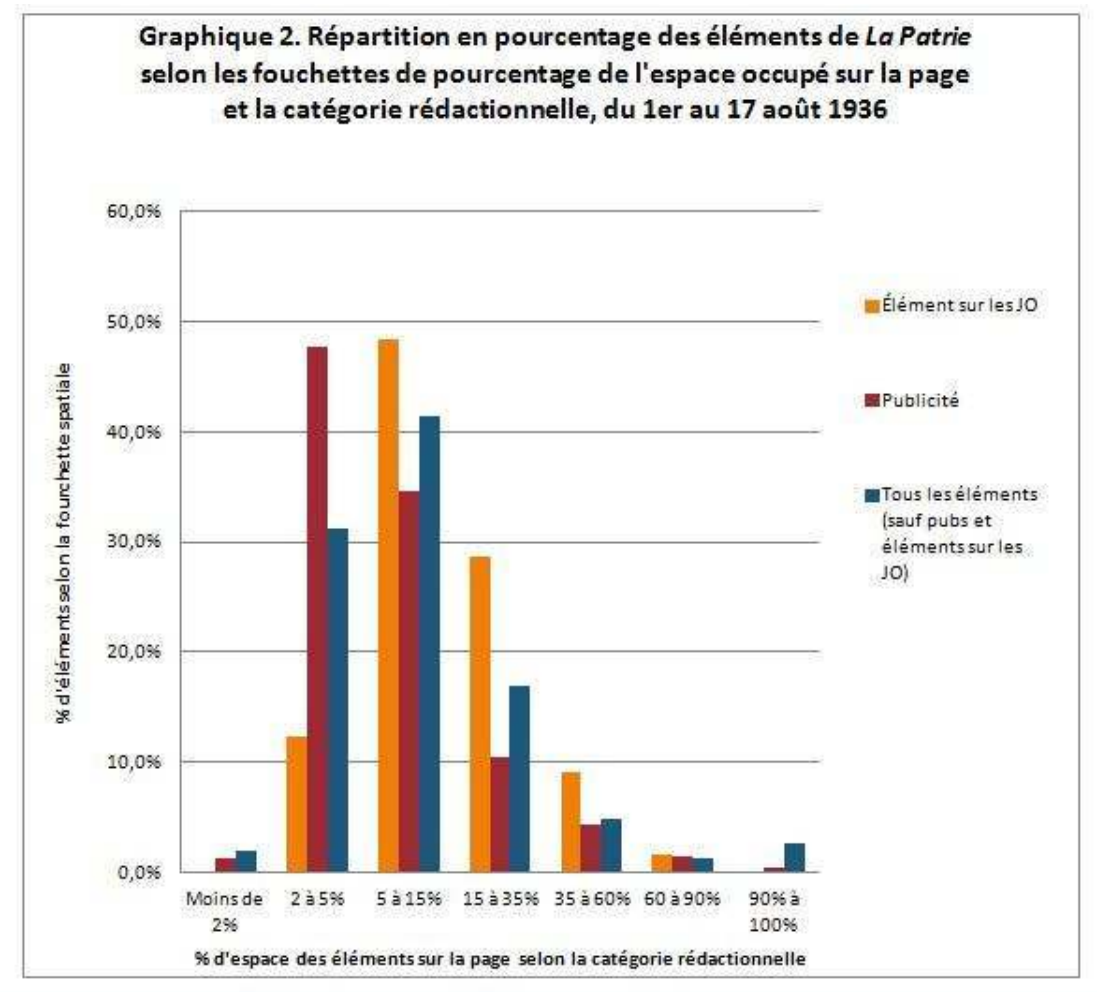


Tableau 2. Distribution des éléments sur les Jeux olympiques en pourcentage selon le type d'illustration et le journal, du $1^{\text {er }}$ au 17 août 1936

\begin{tabular}{|l|c|c|c|c|}
\hline Type dillustration & Montreal Daily Star & La Patrie & La Canada & Lillustration Nouvelle: \\
\hline Dessin & $0,0 \%$ & $1,3 \%$ & $0,0 \%$ & $0,0 \%$ \\
\hline Motifs typographique & $0,0 \%$ & $1,3 \%$ & $0,0 \%$ & $0,0 \%$ \\
\hline Photo-action & $2,6 \%$ & $7,8 \%$ & $2,6 \%$ & $13,2 \%$ \\
\hline Photo-portrait & $3,9 \%$ & $11,7 \%$ & $2,6 \%$ & $15,8 \%$ \\
\hline Aucune illustration & $93,5 \%$ & $77,9 \%$ & $94,9 \%$ & $71,1 \%$ \\
\hline Total & $100,0 \%$ & $100,0 \%$ & $100,1 \%$ & $100,1 \%$ \\
\hline
\end{tabular}
utilisé la photographie (photo-portrait et photo-action ${ }^{32}$ ) dans respectivement $19,5 \%$ et
$29 \%$ des éléments portant sur les Jeux. Cela n'a rien d'étonnant, ces deux journaux étant largement illustrés. Cependant, dans L'Illustration, la proportion d'images dans les articles liées aux JO est quantitativement plus importante que celle que l'on retrouve dans le reste du journal ( $29 \%$ contre $18 \%$ ). Dans le cas du Star et du Canada, les photos et les autres supports visuels demeurent marginaux, ce qui correspond là encore aux pratiques usuelles de ces journaux. En somme les journaux qui utilisent largement les illustrations se servent des photos et des photos-vignettes dans une proportion égale ou supérieure à leur pratique habituelle dans le reste du journal ; ceux qui n'en usent pas habituellement, ne le font pas davantage pour les JO. À Montréal, les Jeux ne sont donc pas un événement «photographique ${ }^{33} »$.
Tableau 3. Répartition des éléments sur les Jeux olympiques de Berlin selon la nature et le journal, du $1^{\text {er }}$ au 17 août 1936

\begin{tabular}{|l|c|c|c|c|}
\hline Nature: & Montreal Daily Star & La Patrie & te Canada & Lillustration nouvelle \\
\hline Chronique & 27 & 10 & 1 & 0 \\
\hline Éditorial & 1 & 0 & 0 & 0 \\
\hline Grand titre & 0 & 6 & 1 & 1 \\
\hline Nouvelle & 134 & 48 & 29 & 22 \\
\hline Photo-vignette & 13 & 17 & 2 & 19 \\
\hline $\begin{array}{l}\text { Publicité } \\
\text { Rubrique }\end{array}$ & 0 & 1 & 1 & 0 \\
\hline $\begin{array}{l}\text { Liste (horaire, résultats, } \\
\text { etc.) }\end{array}$ & 16 & 13 & 3 & 34 \\
\hline Total & 232 & 125 & 71 & 76 \\
\hline
\end{tabular}

Les éléments sur les JO apparaissent au premier coup d'œil dépourvus de subjectivité : « Nouvelle», «Liste» et «Photo-vignette» dominent (voir Tableau 3). Pourtant nous remarquons que les chroniques et les éditoriaux sont nombreux. Près de $15 \%$ des chroniques publiées durant la période mentionnent les JO, ce qui contraste avec le faible pourcentage d'éléments liés aux Jeux dans la couverture générale (entre 1,8 et 3,9\%). Notons le contraste entre le Star et La Patrie d'une part, Le Canada et L'Illustration nouvelle d'autre part. Tandis que La Patrie, et surtout le Star, consacrent de nombreuses chroniques aux Jeux ${ }^{34}$, L'Illustration nouvelle qui n'en publie aucune, privilégie un traitement par l'image avec 19 photos-vignettes. Le Canada publie une seule chronique partiellement consacrée aux Jeux et deux photos-vignettes : $50 \%$ des éléments consacrés aux Jeux y 
sont des listes. Il faut remarquer que toutes ces chroniques sont placées dans les pages sportives, lieu où les objets "politique » et "guerre/militaire " ne sont virtuellement jamais présents. Ajoutons enfin qu'aucun reportage identifié comme tel n'est publié, ce qui est congruent avec les analyses que nous avons menées lors de dépouillements par échantillonnage (semaine de journal recomposée) de huit journaux montréalais de l'entre-deux-guerres ${ }^{35}$.

17 Enfin, globalement, les JO se classent parmi les dix objets ${ }^{36}$ le plus souvent traités dans le Star ( $9^{\mathrm{e}}$ rang), La Patrie ( $9^{\mathrm{e}}$ rang) et L'Illustration nouvelle ( $\left.10^{\mathrm{e}} \mathrm{rang}\right)$. Dans Le Canada, ils se classent au $14^{\mathrm{e}}$ rang. En proportion, le Star est le journal dans lequel nous retrouvons la plus importante présence de l'objet JO (3,9\% du contenu). Pour les trois autres journaux, cette proportion varie entre 2,8 et $1,8 \%$ du contenu. Dans tous les cas, la couverture des Jeux se retrouve loin derrière la politique (de $19 \%$ à $12 \%$ du contenu), ou l'économie et les finances (16\% à $9 \%$ ). De plus, de manière prévisible, compte tenu du fait que $93,2 \%$ de tout ce qui est relatif aux JO se retrouvent dans la section des sports, dans tous les journaux, à l'exception de La Patrie ${ }^{37}$, plus de $90 \%$ des éléments traitant des Jeux sont explicitement associés à l'objet sport.

18 L'ensemble des chiffres que nous venons d'exposer nous permet de tirer une première conclusion quant à la couverture des Jeux de Berlin. D'une part, si l'événement a généré une couverture continue sur l'ensemble des deux semaines qu'ont duré les compétitions, celle-ci n'a pas engendré suffisamment de contenu pour concurrencer la couverture réservée à des sujets comme la politique ou l'économie. De plus, malgré les circonstances politiques entourant les Jeux, c'est sous l'angle sportif que l'événement a été majoritairement traité, dans les formes qui sont celles qui dominent traditionnellement dans les pages sportives. Remarquons aussi que la fréquence des types de texte les plus propices à l'expression d'une opinion (éditorial et chronique, principalement) varie selon les périodiques, le Star se démarquant par l'abondance de ses chroniques. En résumé, les Jeux olympiques sont présentés comme un événement sportif majeur, mais qui n'engendre pas une couverture hors norme. Le traitement journalistique des Jeux s'apparente, avec quelques nuances, à ce qui se trouve dans le reste des pages des journaux analysés, adoptant les pratiques usuelles du journalisme d'information de tradition nord-américaine, dans lequel textes d'information, textes d'opinion et publicité sont en principe distincts.

\section{Modalités énonciatives : pauvreté des sources et contrôle de l'information}

19 Un regard sur les modalités énonciatives des textes portant sur les Jeux permet d'approfondir ce premier coup d'œil et de nuancer l'interprétation. Nous avons, dans le cadre de cette seconde partie de notre étude, ajouté aux résultats issus de l'analyse systématique précédemment évoquée l'étude longitudinale des sept autres journaux dépouillés.

Nous avons relevé de façon systématique les sources explicites - agences de presse ou journaux. Nous cherchions à déterminer les sources externes les plus souvent employées de manière à identifier les recoupements entre les textes. Nous avons aussi relevé le nom des villes d'où provenaient les articles - généralement Berlin et occasionnellement Grünau ou Kiel, autres localités allemandes où se déroulent des compétitions -, ce qui 
révèle que les informations sur les Olympiques arrivent quasi exclusivement d'Allemagne sans médiation ${ }^{38}$.

Dans les journaux, tant anglophones que francophones, l'agence de presse la plus exploitée en ce qui a trait aux Jeux est la Presse Canadienne ${ }^{39}$. Celle-ci fournit au Star plus du quart de ses informations. Le Herald et la Gazette s'appuient aussi de manière importante sur le fil de presse de cette agence. Dans La Patrie et Le Canada, le contenu provenant de la Presse Canadienne compte respectivement pour $21 \%$ et $7 \%$. Les journaux anglophones utilisent également la Presse Associée ${ }^{40}$ - dont le Star dans une proportion de $23 \%$, La Presse et le Herald puisent aussi de nombreux articles dans le fil de la United Press $^{41}$. Le Keneder Odler a quant à lui accès à une agence de presse dont nous n'avons pas trouvé trace dans les autres journaux, la Jewish Telegraphic Agency ${ }^{42}$, tout en tirant aussi des informations de la Presse Associée (mais pas de la Presse Canadienne). Cependant, la provenance de l'information est absente de nombreux articles ${ }^{43}$. Ainsi, un peu plus de $75 \%$ des éléments portant sur les Jeux dans La Patrie n'ont pas de source explicite et $47 \%$ dans Le Canada. L'information sur les Jeux présentée dans Le Devoir ${ }^{44}$ et l'Illustration nouvelle n'est jamais explicitement imputée aux agences de presse : il faut imaginer pourtant que ces articles, comme ceux de La Patrie, sont des collages ou des réaménagements de ce qui paraît dans d'autres journaux ou est diffusé à la radio.

Par ailleurs, vérification faite, aucun journal montréalais n'a dépêché de reporter accrédité aux Jeux ${ }^{45}$. Les journaux montréalais sont donc presque exclusivement tributaires des envois des agences de presse reçus depuis l'Allemagne ${ }^{46}$. Or celles-ci sont soumises aux filtres imposés par le régime nazi. Reproduit dans plusieurs journaux ${ }^{47}$, un article du London Daily Herald traite de la surveillance des journalistes internationaux par la «police secrète» et révèle les pratiques de censure des organisateurs olympiques allemands : « Les journalistes ne parlent plus. Lorsqu'ils sont forcés de parler, ils le font à voix basse. Chaque homme est soupçonnét8 ${ }^{4}$. Il est donc raisonnable de penser que les journalistes des agences de presse censurent leurs propos et que, conséquemment, les journaux sont limités à une couverture évitant les polémiques, une couverture neutralisée en quelque sorte ${ }^{49}$. Dans ce contexte, les correspondances exclusives «spécial au Petit Journal», «correspondance spéciale [à L'Autoritée » et les lettres de lecteurs portant signatures reçues depuis l'Europe revêtent, malgré leur rareté, une importance déterminante. Ce n'est sans doute pas un hasard si l'unique «Correspondance spéciale » de l'Autorité, anonyme, porte sur la censure et sur la « trêve à la barbarie » que représentent les $\mathrm{J}^{50}$ - thème absent de la couverture toute sportive des agences.

23 À ce problème de censure s'ajoute la pauvreté relative des sources en termes de diversité. Alors que pour les autres sujets traités dans les journaux, nous observons une grande variété de sources, trois agences de presse seulement sont à l'origine de la majorité des articles sur les JO. Nous notons entre autres la quasi-absence de l'agence Havas et de la British United Press, des agences importantes, largement utilisées habituellement. Les agences peuvent, bien sûr, avoir conclu entre elles des ententes d'exclusivité. Néanmoins les sources étant souvent les mêmes d'un journal à l'autre, leur lecture laisse une impression d'homogénéité un peu troublante. Les Jeux donnent en somme à entendre fort peu de voix différentes.

Toutefois, la sélection et la manipulation de l'information peuvent contribuer à réduire cette homogénéité en introduisant une forme de différenciation des discours. Pensons notamment aux effets du tri effectué à partir des fils de presse. Même lorsque les événements sont traités sur un ton neutre, informatif, qui évite la prise de position 
directe, ils peuvent inviter à la controverse. Le seul fait de choisir d'y consacrer quelques lignes ouvre un espace polémique qui déconstruit l'univocité du discours. Les événements controversés qui ont fait l'objet d'articles informatifs, même brefs, sont nombreux: le renvoi d'Eleonor Holm Jarret de l'équipe américaine à la suite de sa consommation d'alcool jugée excessive pendant la traversée ${ }^{51}$; la « suspension » du coureur Jesse Owens de l'Union Athlétique Amateur ${ }^{52}$; le comportement des leaders allemands devant la supériorité des athlètes noirs américains ${ }^{53}$; le départ de la délégation péruvienne à l'issue d'un match de soccer ${ }^{54}$; la remise en question du sexe de la championne américaine Helen Stephens ${ }^{55}$; la méthode de pointage au classement des nations ${ }^{56}$; l'implication insuffisante de l'État canadien dans le sport olympique ${ }^{57}$. On remarquera que ces sujets renvoient tous à des questions de race et de genre ou aux relations entre nation et sport. Même neutre, privée d'intervention de la part de l'énonciateur, la couverture des Jeux tirée des fils de presse aborde indirectement le point aveugle des Jeux, la nature du régime en place à Berlin et les enjeux liés à la race, au genre et à la nation qui lui sont associés, directement ou indirectement ${ }^{58}$.

Autre cas de figure. Lors de la traduction et du découpage des textes des agences, certains aspects peuvent être retenus ou écartés par les différents journaux. Ainsi, dans son bilan des Jeux, Le Canada consacre un paragraphe complet aux succès de Jesse Owens. L'Illustration Nouvelle, dont le rédacteur en chef, Adrien Arcand, est connu pour son adhésion au fascisme et ses publications ouvertement fascistes et antisémites ${ }^{59}$, reprend l'essentiel de l'article, mais ne fait cependant aucune mention de l'athlète noir américain, pourtant considéré comme la vedette des Jeux ${ }^{60}$. Un autre article paraît dans L'Illustration du 6 août ${ }^{61}$, il est tiré de la United Press et de la Presse canadienne, comme le révèle la comparaison avec des articles du Star (UP 5 août ${ }^{62}$ ), de La Patrii ${ }^{63}$ et du Canada ${ }^{64}$ (PC 6 août), mais L'Illustration nouvelle reprend seulement certains des éléments des textes originaux, ce qui modifie la perception de l'événement. On peut y lire qu'Owens « semblait fatigué et guère heureux » pendant la remise des médailles et qu'il ne « souria [sic] pour la première fois, [que] lorsqu'on lui offrit un imperméable $\aleph^{65}$. Dans Le Star, qui reproduit un commentaire de UP sur la cérémonie de remise des médailles du 200 mètres, on trouve l'explication - «a downpour of rain drenched the main olympic stadium and chased many of the 65,000 spectators ${ }^{66} "$ - que L'Illustration oblitère, rendant Owens peu sympathique.

Notre dernier exemple concerne la manipulation des images: dans La Presse une photo donne clairement à voir le dessin de la croix gammée sur le bras d'Hitler (fig. 1). Dans L'llustration la croix gammée est tronquée, ce qui la banalise mais invite le lecteur à compléter l'image (fig. 2). Dans La Patrie, en page 2, le cadrage de la même photo efface la croix gammée (fig. 3). 


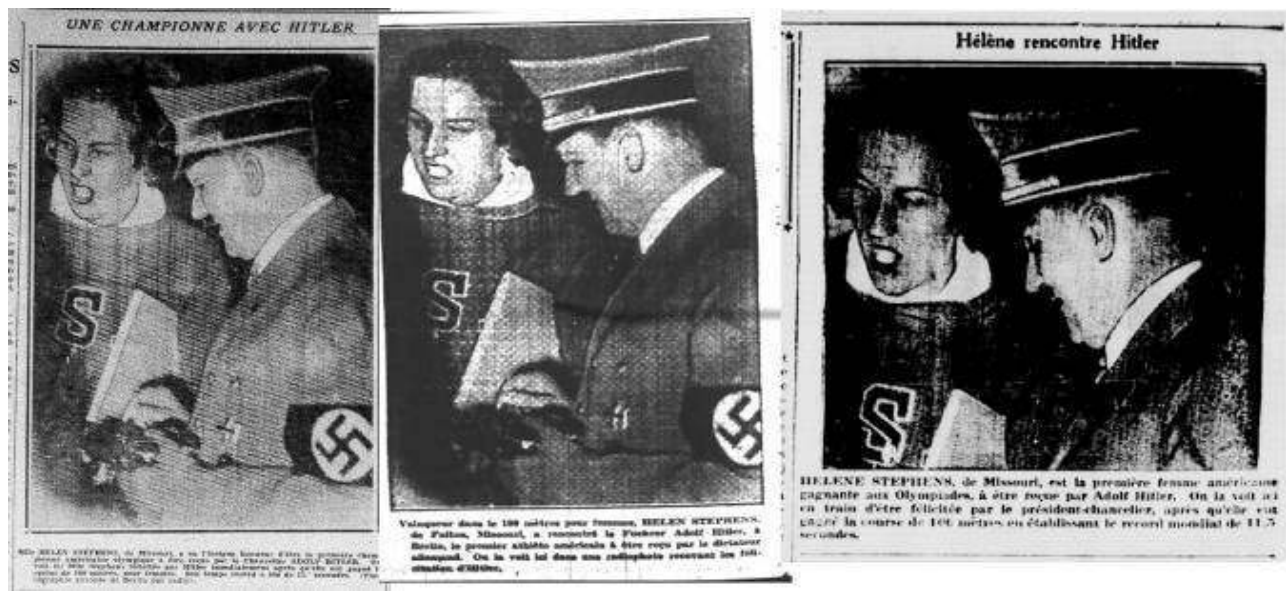

Fig. 1 : La Presse, 5 août 1936, p. 16 ; Fig. 2 : L'Illustration nouvelle, 8 août 1936, p. 24 ; Fig. 3 : La Patrie, 7 août 1936, p. 2 textes non signés qui racontent les événements sans émarger explicitement à des dépêches d'agence de presse. Ceux-ci semblent, d'une certaine façon, prolonger les tableaux de résultats qui occupent une grande surface des pages sportives. Le Canada, L'Illustration nouvelle, La Patrie et $\mathrm{La}$ Presse y maintiennent ainsi une certaine distance à l'égard des événements. Celle-ci est perceptible à travers l'usage nettement dominant qui est fait, dans tous les journaux, de la troisième personne du singulier, ce qui place l'énonciateur hors du récit. L'emploi d'expressions hyperboliques convenues telles que «l'invincible Jesse Owens ${ }^{67}$ », « les poissons-humains japonais ${ }^{68}$ » ou le « coureur le plus rapide de l'univers ${ }^{69} "$, qui reviennent dans la description des performances des athlètes gagnants, ne parvient pas à personnaliser le discours. Ces stratégies rhétoriques un peu maladroites, qui relèvent du cliché, se fondent dans la neutralité générale de la couverture.

Quelques textes rompent cependant avec le tout-venant de la couverture. La Patrie affiche, à l'occasion, dans certains des entrefilets des rubriques non signées que sont 
"Potins de Berlin » et "Réflexions brèves ${ }^{70}$ ", respectivement placées dans la page des sports et dans la page éditoriale, un ton ironique, rappelant l'existence de polémiques hors du ronron des médailles et des tableaux d'honneur. Ainsi dans les «Réflexions brèves» du 5 août, on peut lire: "Afin d'y mettre de la nouveauté, la prochaine Olympiade devrait être consacrée aux sports professionnels, ce qui permettrait d'inscrire au programme le grand sport de la guerre » et dans les « Potins de Berlin » du 6 août, on mentionne que «Mlle Stephens porte des souliers, pointure $\mathrm{N}^{\circ} 11^{71}$ » après la remise en question de son sexe dans un journal polonais.

31 Autre rupture de ton, l'hyperbolisation qui marque toute nouvelle concernant les grandes vedettes des Jeux dans L'Illustration et La Patrie. Qu'il s'agisse de Holm Jarrett, de Stephens ou d'Owens, des photos sont publiées, des aspects de leurs vies personnelles et des scandales qui les touchent sont étalés. La création d'un vedettariat olympique détourne, à coup d'épithètes et de métaphores, l'attention des réels jeux de pouvoir qui sous-tendent les controverses, tout en faisant vendre plus de copies. Aussi La Patrie, le deuxième journal en importance pour le nombre d'éléments reliés aux Jeux, rend-elle compte systématiquement de toutes les polémiques sur un ton suscitant la curiosité ou la stupéfaction plutôt que la réflexion ou la prise de position, liant vedettisation et sensationnalisme. Les « nouvelles » sont ainsi livrées sans analyse, sans jugement. Il en va de même du traitement que La Presse réserve à certaines cérémonies comme celle durant laquelle le Canada restitue à l'Allemagne des débris d'avions militaires abattus durant la guerre de 14-18. Cette "impressionnante cérémonie» ne bénéficie d'aucune contextualisation : l'information, curieusement placée dans la page des sports, est livrée de manière brute ${ }^{72}$.

Pourtant, La Patrie et La Presse voient, comme plusieurs autres journaux, que le sport pratiqué dans un contexte patriotique est le reflet d'importantes luttes quant à la vision de la nation. La récurrence de symboles nationaux canadiens comme la Feuille d'érable, l'insistance mise sur l'origine des athlètes - «Edwards de Montréal ${ }^{73}$ ", ou « Frank Amyot d'Ottawa ${ }^{74}$ ", l'évocation régulière d'institutions canadiennes comme l'Université McGill témoignent de cet investissement national. Cette dimension nationale est explicitée dans certains articles de La Patrie, du Canada et du Star, quand la première personne du pluriel ou le nom du pays fait irruption, dans des titres de rubriques par exemple: "Nos espérances aujourd'hui ${ }^{75}$ », « Nos inscrits aujourd'hui ${ }^{76}$ ", « Nos gloires et leurs exploits ${ }^{77}$ ", « What Canada Did at Olympic Today ${ }^{78}$ », « How Canadians Fared Yesterday ${ }^{79}$ », « Les chances du Canada aujourd'hui ${ }^{80} "$, ce qui accentue l'engagement du scripteur et crée une connivence avec le lecteur, les deux faisant front avec les athlètes canadiens. L'emploi du "nous » ou de pratiques assimilées, comme la réduction de la couverture à certains athlètes ou à des athlètes d'une nationalité particulière, contribue à la création d'une communauté de lecteurs, et peut en effet insuffler aux textes un certain enthousiasme au sein duquel l'avenir de la collectivité croise l'axe des Jeux. C'est dans la définition de ce «nous» que les journaux, voire les chroniqueurs, se distinguent. Il peut s'agir d'un «nous » canadien-français, d'un "nous » britannique, d'un "nous » anglo-canadien, voire italo-canadien, lorsque L'Italia, par exemple, couvre quasi exclusivement les résultats canadiens et italiens. Le "nous " américain est aussi présent, dans le Star, qui publie de longs textes d'Henry McLemore (UP) dans lesquels se trouve affiché un nationalisme américain au chauvinisme impudent ${ }^{81}$.

Il arrive aussi que les conflits sous-jacents entre ces diverses appartenances soient explicités. Ainsi, dans la Revue moderne (une revue familiale qui possède une image forte 
de soutien à la culture canadienne-française, comme on l'appelle à l'époque), le seul texte portant sur les Jeux est écrit au "nous ${ }^{82}$ ». Détaché du déroulement des Jeux, il est essentiellement centré sur la politique nationale canadienne: les conflits entre « l'élément français du Canada » et le gouvernement fédéral y paraissent avivés par la tenue des Jeux et le « nous » est critique, qui s'oppose au « on » du pouvoir fédéral :

Nous ne regrettons qu'une chose, c'est que l'élément français du Canada ne soit pas plus largement représenté à Berlin. On n'a pourtant pas hésité à le recruter, cet élément négligeable, lorsqu'il s'est agi d'aller se faire démolir sur les champs de bataille ${ }^{83}$.

Le choix d'un cadre énonciatif consacrant la distance entre l'énonciateur et son objet ou, au contraire, visant à l'abolir, en ralliant une collectivité nationale, apparaît comme une composante importante du ton des textes, un trait qui marque la couverture et y introduit des tensions interdiscursives.

\section{Modalités énonciatives : signatures et lieux du jugement}

35 La signature est, du point de vue énonciatif, un geste fort qui suppose une forme d'engagement et l'exercice minimal du jugement. Elle est rare dans notre corpus et presque exclusivement apposée à des lettres ou des chroniques. Mais, parce qu'on y trouve clairement énoncés des jugements, il convient d'ajouter à cette liste les correspondances spéciales non signées, de même que les éditoriaux, non signés au Canada à l'époque, qui expriment la ligne éditoriale d'un journal. C'est là bien sûr que l'on trouve les énoncés qui dépassent la couverture anecdotique et inscrivent les Jeux dans des débats sociaux et politiques. Ce qui caractérise ces divers objets est leur circulation hors du discours homogénéisé des agences de presse, voire hors des pages sportives.

Abordons d'abord les textes qui se présentent comme des témoignages directs à propos des Jeux.

Les articles émanant de chroniqueurs des agences de presse depuis Berlin sont majoritairement factuels et strictement sportifs, à l'exception de ceux de Henry McLemore, qui adopte la forme du reportage, se dépeignant comme un témoin qui raconte ce qu'il a vu. Il ridiculise les Européens qui ne comprennent pas le baseball ${ }^{84}$; il relate par le menu une séance d'haltérophilie sans rien omettre du déshabillage et de la pesée d'athlètes présentés comme bestiaux ${ }^{85}$; il se moque du Fuhrer («Brother Hitler ») et de ses gardes qui demeurent debout sans bouger ${ }^{86}$. Manifestement, ses chroniques ont échappé à la censure en vigueur.

D'autres journaux publient des textes qui se présentent comme des « correspondances ». L'Autorité, qui ne couvre pas les Jeux, fait paraître la «correspondance spéciale [à L'Autorité] » dont il a été question plus haut. Le Petit Journal, qui traite fort peu des Jeux, consacrant ses pages sportives au sport professionnel, publie trois textes et une photo " exclusive » d'un champion, Frank Amyot ${ }^{87}$, signés "spécial au Petit Journal », anonymes donc. Si le ton y est didactique à propos de l'organisation des Jeux, de la réalisation du vélodrome et du bilan final; un long développement à propos de la propagande nazie, publié dans l'article sur l'ouverture des JO, le 2 août, donne à voir une réflexion inquiète à propos de la "césure dans le cours des événements» que constituent les JO «pour les politiciens du monde en général » et surtout de ce qui suivra « [a] près les JO ». Le Keneder 
Odler, qui ne couvre pas du tout le sport, publie une correspondance envoyée de Paris signée L. Berek, qui expose la délicate situation du gouvernement socialiste de Léon Blum en France à l'égard du boycott, d'abord prêché dans plusieurs cercles gauchistes, désormais devenu un sujet tabou ${ }^{88}$. Pour ces journaux, il est clair que les JO ne sont pas seulement un événement sportif.

Dans les autres journaux, les chroniques sportives signées par des collaborateurs réguliers, bien connus du public, constituent quantitativement le lieu principal de l'exercice du jugement. Malgré leur faible proportion dans l'ensemble du corpus sur les Jeux, l'importance de ces chroniques est manifeste sur le plan de la mise en page (filets, ornementation typographique, photo du chroniqueur, choix de l'espace) (fig. 4, 5, 6, 7, 8, 9).

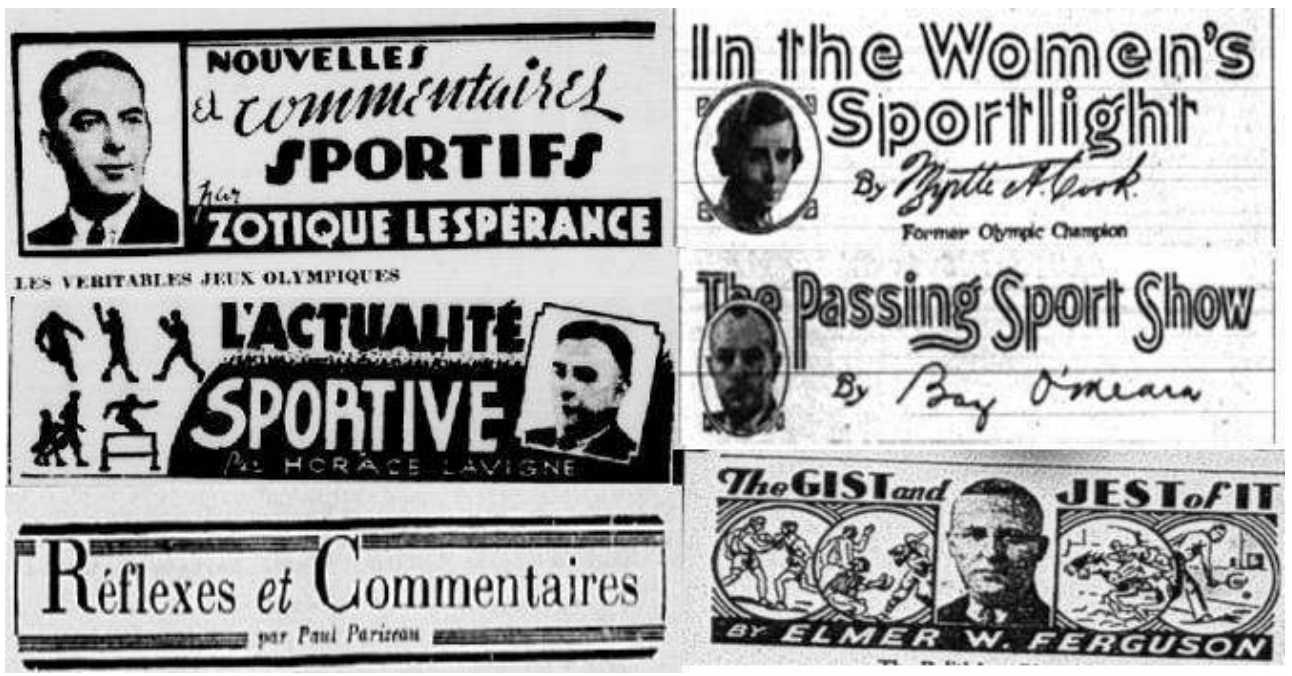

Fig. 4 à 9 : (Du haut vers le bas) Zotique Lespérance et Horace Lavigne écrivent dans La Patrie, Myrtle Cook et Baz O'Meara dans le Star, Paul Parizeau dans Le Canada et Elmer Ferguson dans le Herald.

De plus, la transmission d'idées y bénéficie de l'autorité d'une signature qui fait manifestement partie de la stratégie de vente des journaux ${ }^{89}$. Ainsi, La Patrie compte dans ses rangs deux chroniqueurs sportifs, Zotique Lespérance et Horace Lavigne, de même que le chroniqueur radio George Galipeau; tous trois s'intéressent aux Jeux olympiques à au moins deux reprises ${ }^{90}$. Myrtle Cook, une ancienne médaillée olympique canadienne, et Baz O'Meara sont les deux chroniqueurs sportifs du Star, comme Elmer W. Ferguson du Herald et Dunc McDonald de la Gazette. Ils incorporent à leurs chroniques régulières des informations sur les JO. Dans le Canada, une seule des chroniques de Paul Parizeau porte sur les Jeux. Celui-ci y dénonce le soutien financier des universités américaines à l'endroit des athlètes amateurs et commente le comportement d'Hitler :

Fait plutôt insolite, chaque fois qu'un athlète de race noire a décroché les honneurs d'une épreuve, Hitler a brillé par son absence, laquelle a été motivée par un appel urgent ou quelqu'excuse aussi diplomate. Voilà une vérité qui en dit beaucoup sur le mécontentement que doit ressentir le Fuehrer [sic] en constatant que les nègres américains ont enlevé à l'Allemagne cette suprématie à laquelle elle aurait pu aspirer sans eux ${ }^{91}$.

Disposant d'un statut qui leur confère une certaine liberté de ton, les chroniqueurs utilisent tous cette liberté pour aborder des questions sociales ou politiques qui débordent le caractère sportif de la compétition, développant de manière plus ou moins explicite, plus ou moins combative, des réflexions liant l'événement sportif à un contexte sociopolitique plus large. Dans le Star, Cook, qui couvre les compétitions féminines, dénonce le 
rôle social domestique dans lequel Mussolini veut enfermer les femmes, en défendant la pratique sportive des femmes d'Italie et d'ailleurs, et O'Meara se prononce explicitement contre l'utilisation de la «théorie des races» dans l'appréciation des performances sportives $^{92}$. Dunc McDonald souligne dans la Gazette l'écart entre les idéaux olympiques et la situation politique explosive en Europe ${ }^{93}$. Zotique Lespérance écrit que «Les épreuves sur piste et pelouse sont le seul domaine sportif où l'Américain traite d'égal à égal cette race pour laquelle il démontre, en toute autre circonstance, un véritable mépris ${ }^{94}$ » et il évoque le refus d'Avery Brundage de faire concourir des athlètes juifs à Berlin pour plaire au régime nazi ${ }^{95}$.

Scaramouche, dans L'Autorité, profère des récriminations virulentes au sujet du régime nazi, se livrant à la déconstruction de la théorie des races ${ }^{96}$ et A. S. du Keneder Odler ${ }^{97}$ attaque l'organisation olympique, dénonçant vertement le gouvernement allemand: " "Goebbels a commandé aux Juifs allemands d'accueillir les invités étrangers des Olympiques avec un sourire." Que sais-je! Goebbels ne va tromper personne. Les Juifs pourraient aussi forcer un rire ${ }^{98} »$. Remarquons que ces deux chroniqueurs n'apparaissent pas dans la page sportive. Cependant, tous les chroniqueurs qui abordent frontalement la situation internationale explosive et les caractéristiques du régime nazi présentent les JO comme s'inscrivant dans une stratégie politique dont les enjeux moraux doivent être explicités.

C'est aussi, à sa manière, ce que fait Herblock, dans une caricature reproduite par le Herald $^{99}$, en liant métaphoriquement la flamme de la Guerre d'Espagne et celle des Jeux, dans un dessin où l'on voit, en gros plan, un guerrier transportant la flamme olympique. Au-dessus de sa tête, est inscrit "The War Spirit: The Flames of Civil Strife in Spain". Beaucoup plus petit (il lui va à peine à la cheville), un athlète porte la flamme olympique et un cartouche indique: "The Olympic Spirit ${ }^{100}$ ». De façon nette, "l'esprit guerrier » écrase l'« esprit olympique »... (fig. 10).

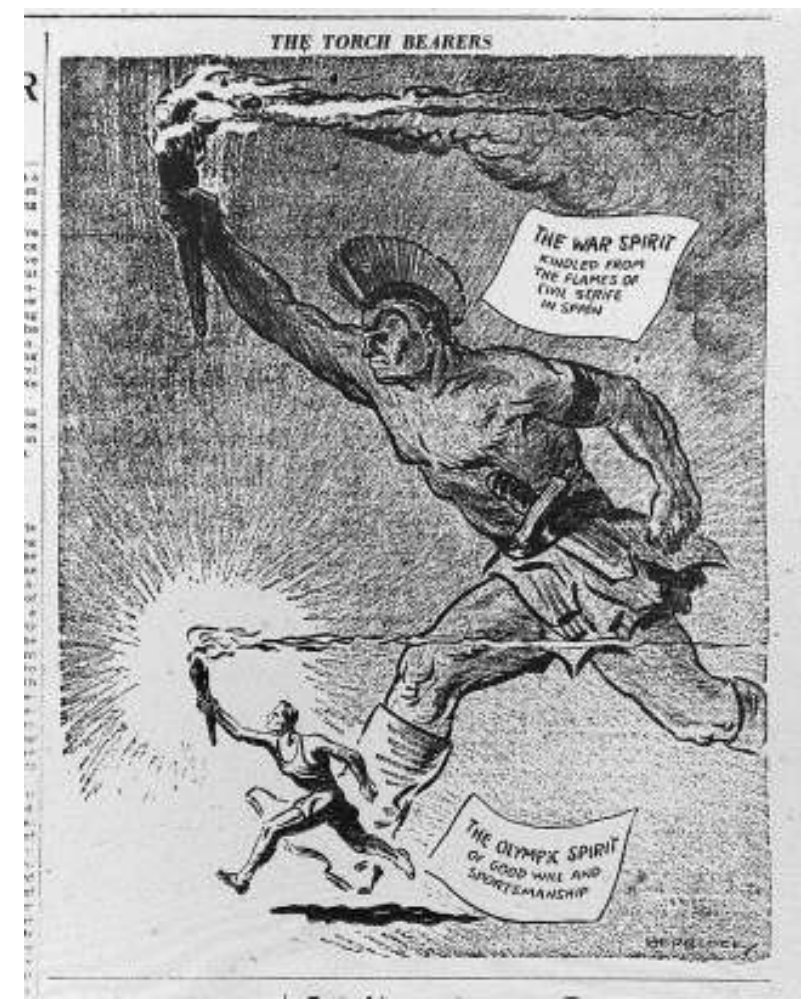

Fig. 10 : Herald, 10 août 1936, p. 4. 
naïveté non plus dans les éditoriaux parus dans La Presse, le Keneder Odler et le Star, lesquels expriment une position claire contre le régime nazi et critiquent l'instrumentalisation des JO. Le 3 août, les éditoriaux du Star et du Keneder Odler sont partiellement consacrés aux JO ${ }^{101}$. Remontant à 1918, l'éditorialiste du Star explique que les nazis jugent que l'échec allemand de la Grande Guerre est dû à une "mauvaise publicité » et qu'ils entendent utiliser les Jeux pour rectifier leur mauvaise image. Les « Nouveaux Allemands» sont présentés comme des «maîtres de la propagande » et le Canada comme un pays qui s'est laissé embrigader (« embroiled in the issue ») dès les Jeux de Garmish Partenkirchen, lorsque ses athlètes ont utilisé un salut proche de celui des nazis. Le texte se termine sur une remarque d'ordre moral: les victoires d'Owens, Jackson et Edwards apportent un déni aux théories sur la supériorité de la race " nordique ", évoquée de manière « disgracieuse » lors de la Cérémonie d'Ouverture par Theodor Lewald, président du comité organisateur des Jeux. Cela, écrit l'éditorialiste sur un ton sarcastique, devrait être "murmuré à l'oreille des théoriciens de la supériorité Nordique et aux membres du Klu Klux Klan International » (notre traduction). Le Keneder Odler, qui expose régulièrement les exactions nazies ${ }^{102}$ et dénonce les Jeux comme manœuvre visant à distraire les Occidentaux, définit les JO comme une " Mascarade », ironisant sur l'exercice de propagande qui vise à faire des nazis des gens " agréables » et de leur terre " un paradis $»^{103}$. Le 4 août, dans La Presse, les Jeux olympiques sont l'un des quatre thèmes de l'éditorial ${ }^{104}$. Présentés comme répondant à un objectif intérieur développer chez les Allemands le «culte des exercices corporels" - et à un objectif extérieur - «prêcher au monde la vertu de l'Évangile nazi » - les JO sont explicitement ramenés à un exercice de propagande. Pour l'éditorialiste les visiteurs qui sont "édifiés ", sont victimes des propagandistes nazis : "Il n'y aurait rien de surprenant, comme on le dit déjà, à ce que, aussitôt les étrangers partis, les persécutions d'autrefois reprennent de plus belle et que le Reich reprenne son vrai visage ». Le ton est grave, indigné.

Les éditoriaux font ainsi front commun contre le Reich, ses " théoriciens de la Nordicité » (selon l'euphémisation de Lewald rapportée par le Star ${ }^{105}$ ) et ses « persécutions ». Le Star, davantage sensible aux parentés entre les théories de la suprématie aryenne et celles du Klu Klux Klan, ne rapporte guère les exactions du Reich. Celles-ci sont toutefois quotidiennement affichées dans le Keneder Odler, qui ne s'adresse cependant qu'aux Montréalais qui lisent le yiddish. La Presse aussi y insiste, répétant deux fois le mot "persécutions" et soulignant que celles-ci sont "violentes [et] dirigées contre les catholiques et les juifs ${ }^{106} »$. Les trois textes sont caractérisés par un recours direct ou indirect à un temps long de l'histoire et le jugement moral exprimé ne s'appuie pas sur des événements récents ou ponctuels mais sur les principes, les «théories " dénoncées. De plus, les trois éditorialistes affirment que les Jo sont une opération de propagande et désignent les visiteurs étrangers, et par extension les autres pays du monde, comme ceux que les nazis veulent tromper. Ils s'attribuent ainsi une fonction démystificatrice. Est-il besoin de souligner que, placés hors des pages sportives et investis de l'autorité de chacun des journaux, ces éditoriaux témoignent à la fois d'une juste compréhension des enjeux et de la désillusion à l'égard d'un événement entièrement détourné de sa signification humaniste ? Il est intéressant de noter que l'un des autres sujets traités dans l'éditorial de La Presse est le $645^{\mathrm{e}}$ anniversaire de la Confédération helvétique dont les principes démocratiques sont présentés comme un exemple

au moment où l'on assiste à l'éclosion de tant de dictatures, de gauche comme de droite et que [sic] l'on entend d'innombrables attaques contre les formes actuelles de gouvernement ${ }^{107}$. 
46 situation européenne, là même où, selon les mots de l'éditorialiste de La Presse, s'exerce la
«politique louvoyante » du régime allemand.

\section{Conclusion}

47 Selon nos analyses, la couverture des Jeux de Berlin qu'offrent les différents journaux montréalais se démarque peu de leurs pratiques usuelles. Toutes semblent fortement déterminées par les règles du journalisme nord-américain qui sépare de manière nette les informations (censées être objectives) et les textes d'opinion, porteurs d'argumentations et de jugements. C'est donc principalement dans les chroniques placées dans les pages sportives et dans les éditoriaux que l'on trouve des énoncés qui relient les JO à des enjeux sociaux et politiques plus vastes. Associés à une signature ou à l'expression claire d'un jugement, ces textes s'opposent d'un point de vue énonciatif aux informations factuelles sur les JO, lesquelles sont enfouies dans une accumulation de tableaux et d'anecdotes dont la signification ne va pas de soi. Vus à distance, ces innombrables petits faits ressemblent à du bruit qui entrave ultimement la communication, noyée sous leur masse. Monopolisant le discours, et l'inscrivant dans l'univocité illusoire de chiffres, de rangs et de faits divers dont le sens serait transparent, ces petits faits vrais apportent de l'information tout en scotomisant la conversation démocratique.

C'est ici que la nature variable de la relation qui existe entre les chroniques, les éditoriaux et la couverture factuelle des Jeux dans un même journal révèle toute son importance. Dans le Star, les informations factuelles sur les Jeux et les courts articles portant sur des événements spécifiques se trouvent "cadrés» au plan matériel comme au plan symbolique par les chroniques qui, visuellement, les encadrent (fig. 11).

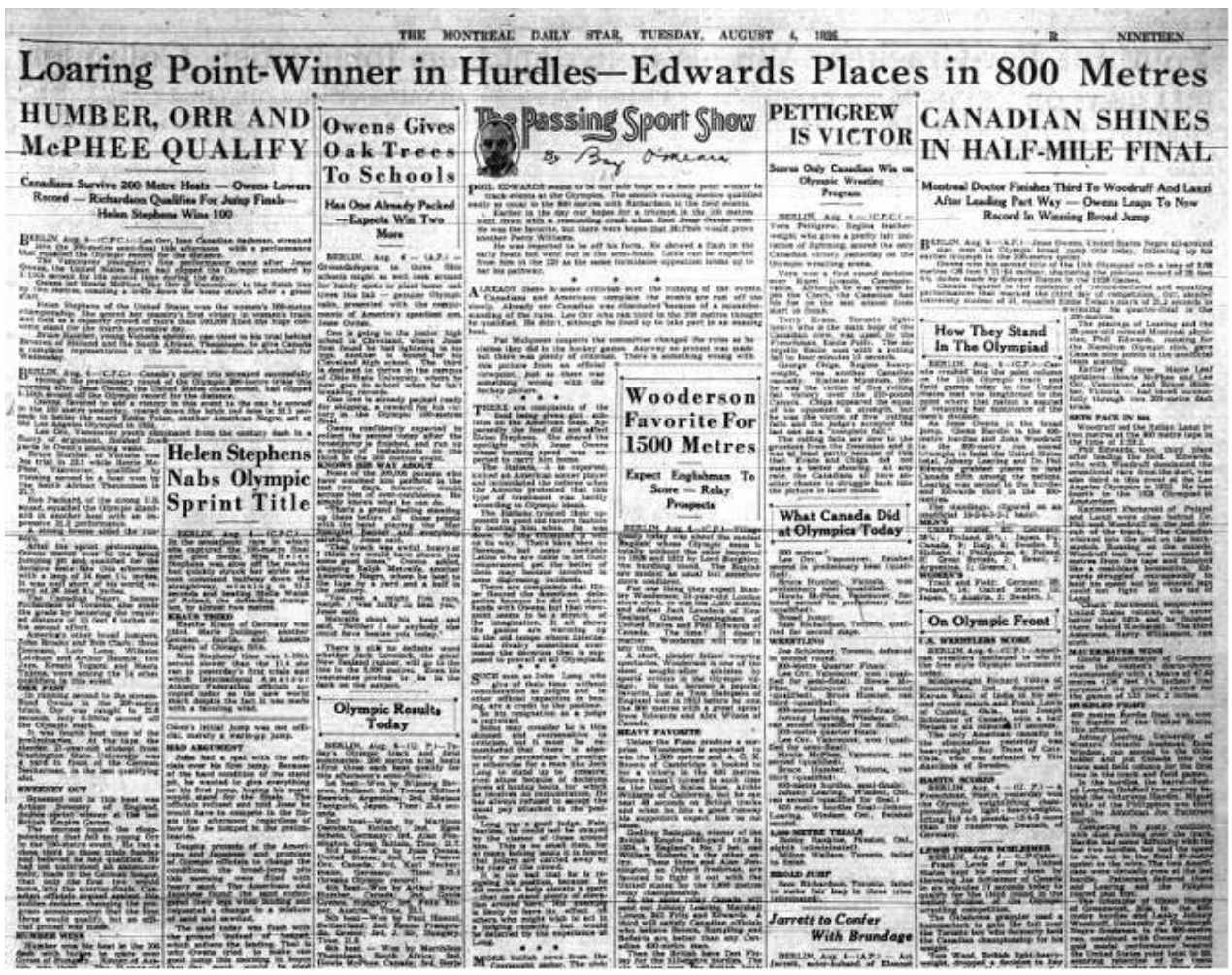

Figure 11 : Vue d'ensemble d'une page de journal, The Montreal Daily Star, 4 août 1936, p. 19. 
s'agit donc d'un véritable cadrage («framing ») de l'information, les chroniques offrant les clés permettant de donner sens aux informations éparses en les inscrivant dans un récit plus général et plus politique. Dans La Patrie au contraire, et malgré certains commentaires de Zotique Lespérance, les informations, dans leur disparate, et en l'absence de jugements ou de commentaires, sont assimilées à du divertissement, ce dont témoigne d'ailleurs l'une des remarques de Lespérance, qui affirme que l'apparition quotidienne d'Hitler dans le stade à partir d'un sous-terrain constituait «l'un des spectacles les plus intéressants » des Jeux ${ }^{108}$. La naïveté apparente de ce commentaire, troublante, témoigne d'une incapacité à exprimer clairement les enjeux politiques des Jeux. Le lecteur qui reçoit ce texte ne peut interpréter les informations sur les Jeux dans le journal, ni a fortiori, les voir comme les signes de l'effort de propagande du régime nazi. Il est ainsi placé dans la position de celui qui, selon les mots de l'éditorial de La Presse , est «victime» des images lénifiantes mises en œuvre et prend une trêve stratégique pour une promesse de paix ${ }^{109}$. L'émiettement des anecdotes, joint à l'absence de point de vue structurant, rend difficile l'exercice du jugement critique de la part du lecteur. Celuici doit composer avec une représentation inerte, impossible à inscrire dans la couverture générale de l'actualité politique.

Parallèlement, il faut convenir que la pauvreté de la couverture des JO dans la presse montréalaise est une réalité. La banalité des images, leur rareté, et leur usage quasi exclusif à des fins de vedettisation des athlètes indiquent bien le manque de perspective esthétique dans le traitement, mais surtout les limites du recours aux agences de presse, canadiennes ou américaines, qui offrent un traitement neutralisé de l'information, en partie lié à l'exercice de la censure nazie. Plus grave, l'absence de reportage, à l'exception de la série de chroniques de Henry McLemore et des textes des correspondants spéciaux du Petit Journal et de L'Autorité, pèse très lourd, contribuant à l'homogénéisation de la couverture. Ce qui frappe dans plusieurs des journaux, comme L'Illustration nouvelle ou Le Canada, c'est l'absence de toute diversité de voix. Ces journaux se font l'écho d'un nombre limité d'agences de presse, de sorte que la diversité des informations est fortement réduite. Si pour expliquer mieux il faut raconter plus, comme le dit en substance Paul Ricœur, l'absence de diversité des sources rend impossible le développement d'un récit complexe des Jeux, dont l'interprétation déborderait la dimension factuelle de l'événement. Les journaux qui gomment leurs sources offrent ainsi un récit lisse qui a statut de vérité ce qui empêche tout investissement critique de la part du lecteur.

On peut voir dans ces conclusions un argument fort en faveur de la diversité des sources, et surtout un rappel de l'importance de la présence de voix singulières prenant la parole à propos des événements. Aujourd'hui, alors que l'un des effets de la concentration médiatique, est que beaucoup de journaux renoncent à envoyer des correspondants couvrir les événements nationaux ou mondiaux et se rabattent sur les agences de presse et des communiqués de nature publicitaire ou dépendante (embedded), réduisant de manière radicale la diversité des voix du journal, les effets de la pauvreté des sources sur la couverture des JO que nous avons observés donnent à réfléchir. Car lorsqu'il n'y a pas de diversité des voix et des sources, pas de lieu dévolu à l'analyse, ni de visée structurante perceptible dans les prises de paroles, le lecteur ne dispose d'aucun outil pour évaluer luimême la qualité de la couverture qui lui est présentée comme le réel. Parmi les journaux examinés, L'Illustration nouvelle est une incarnation extrême de cet enfermement dans un régime de vérité. Il n'y a pas de sources, pas de voix différentes qui s'expriment, aucun lieu n'est dévolu au jugement sur les JO, et le lecteur ne trouve rien qui ressemble à une

Belphégor, 15-1 | 2017 
visée structurante, même implicite, à l'égard de la compréhension des JO. Le journal Le Canada dans une moindre mesure, adopte une même posture. Rappelons que L'Illustration nouvelle est par ailleurs le journal qui manipule le plus les informations, allant jusqu'à effacer les médailles de Jessie Owens de son bilan final. Ce n'est pas un hasard. Nous sommes dans le domaine de la propagande, celle qui repose sur les figures effacées des photos officielles, sur les images manquantes ${ }^{110}$.

Par ailleurs, l'analyse des modalités énonciatives nous a amenés à revoir nos hypothèses de départ, selon lesquelles la couverture des JO varierait principalement selon la langue et le parti politique. Certes, la couverture des JO de Berlin permet de voir à l'œuvre les diverses tensions qui caractérisent le système des journaux montréalais, révélant une organisation complexe des identités «nationales » mises en jeu - dont la présence inattendue d'un "nous" américain dans les pages du Star. Mais la définition des communautés d'appartenance auxquelles renvoient les divers «nous » ne détermine pas tous les enjeux.

En effet, dans le Star, le traitement des compétitions n'est pas anecdotique, ancré qu'il est dans une lecture de la longue durée à l'égard des enjeux politiques et moraux qui agitent le sport olympique, auxquels vient se greffer sans heurt la critique de la propagande allemande. Certes, cela contraste avec les journaux francophones, qui ne couvrent pas vraiment l'athlétisme, et demeurent davantage tournés vers le sport professionnel américain et les ligues mineures locales, ce qui reflète la nature plus populaire de leur lectorat. Cependant, les jugements à l'égard de la mise en représentation des Jeux par le régime nazi et des effets qui en découlent ne sont pas liés à la langue ou à la communauté d'appartenance du journal. En effet, L'Autorité et le Keneder Odler adoptent à l'égard des Jeux une position de principe que partage le Star dans certains éditoriaux. La Gazette, La Presse, le Herald et Le Petit journal, offrent des jugements similaires, même si ceux-ci sont isolés dans le journal. Tous ces journaux articulent leurs critiques autour des mêmes valeurs démocratiques et humanistes, dénonçant avec vigueur la comédie jouée par le régime de Berlin. Il semble donc qu'au-delà des tensions linguistiques et nationales qui organisent les journaux montréalais en un système cohérent, une unanimité relative soit lisible qui fait place au développement de perspectives sociales et politiques à propos des Jeux.

La différence de couverture n'est pas non plus liée aux lignes de parti. Depuis le plutôt conservateur Star jusqu'à la libérale radicale Autorité, en passant par La Presse d'obédience mollement libérale, les journaux qui offrent un lieu de discussion détaché des tableaux des résultats et des anecdotes qui s'apparentent aux faits divers, se rallient en effet contre Berlin, par-delà les lignes de parti. Sans naïveté. Le Canada, pourtant libéral, adopte d'ailleurs une position sans profondeur et le seul commentaire éditorial à propos des Jeux comporte des erreurs factuelles qui semblent liées à la pauvreté des sources ${ }^{111}$.

Il faut aussi noter l'absence de différenciation qui serait liée aux types de journaux desquels ressortissent les périodiques montréalais. Le Star fait, comme La Presse et Le Petit Journal, partie d'une presse d'information moderne axée sur la publicité. Mais L'Autorité est plutôt un journal de parti à l'ancienne, comme Le Canada, tandis que le Keneder Odler qui participe aussi de fait à un système autre, celui des journaux publiés en yiddish à travers le monde, publicise surtout, malgré l'importance qu'y revêtent les nouvelles internationales, les commerces de la communauté, à la manière des journaux locaux. Cela n'empêche pas ces journaux fort différents de réagir de la même façon à ce qu'ils perçoivent comme une menace pour la liberté : la vision du monde nazie. 

sociales qu'ils soulèvent, les Jeux de 1936 ne jouent pas un rôle de catalyseur, provoquant l'ébranlement du système des journaux montréalais déjà fortement mis à mal par la crise économique qui s'est à peine résorbée ${ }^{112}$. En effet, les positions adoptées, principalement dans les éditoriaux et les chroniques, par chacun des journaux, ne peuvent pas être prédites à partir des lignes de fractures autour desquelles s'organise ce système depuis la fin du XIXe siècle: langue de publication, allégeance politique et type de publication, déterminé par le format, la nature du financement et le lectorat visé. L'événement que constituent les Jeux olympiques de Berlin - et ses effets politiques anticipés - aurait ainsi été suffisamment traumatisant pour susciter provisoirement une réorganisation du système des journaux, polarisé autour du degré de conscience que chacun exprime à propos des enjeux soulevés, ce qui annonce les profonds réaménagements qui suivront la Seconde Guerre mondiale. reçue selon laquelle les Québécois auraient été ignorants ou indifférents à l'égard des questions morales liées au régime nazi : celles-ci sont au contraire placées au cœur même $\mathrm{du}$ soubresaut de l'opinion publique que révèlent nos analyses. Il faut convenir que, malgré la faible diversité des voix, les Montréalais ont eu accès à des regards nuancés sur les Jeux et sur les enjeux sociaux et politiques que ceux-ci soulevaient.

Ces conclusions nous rappellent la nécessité de la présence, dans les journaux, de discours comportant des jugements propres à servir de clé d'interprétation aux événements. Les journaux privés de ce type de prise de parole, L'Illustration nouvelle et Le Canada, que tout oppose pourtant (format, orientations, type de circulation, orientations commerciales), réduisent l'événement des JO à une compétition promouvant les valeurs individuelles des athlètes et celles, nationales, d'une nécessaire monstration des forces en concurrence : ils sont à la fois pur divertissement et masque élégant pour le déploiement brut du combat entre nations, par athlètes interposés. Ils offrent à leurs lecteurs une forme perverse de naïveté à l'égard des enjeux sociétaux dans laquelle ceux-ci sont privés de toute participation à la conversation démocratique portant sur les enjeux internationaux.

\section{NOTES}

1. Cette recherche porte sur «La presse montréalaise de l'entre-deux guerres, lieu de transformation de la vie culturelle et de l'espace public ». Nous remercions le Conseil de recherche en sciences humaines du Canada pour son appui.

2. Notre définition du concept de système des journaux s'appuie à la fois sur les travaux de Maurice Mouillaud et sur ceux de Jean de Bonville. Nous y ajoutons le postulat d'une interaction forte entre les journaux, liée à leur coprésence dans un même espace public et à leur appartenance à un discours culturel commun. Dans ce contexte nous faisons donc l'hypothèse que par delà les fractures linguistiques, tous les périodiques étudiés appartiennent à un même système. Maurice Mouillaud, «Le système des journaux (Théorie et méthodes pour l'analyse de 
presse) ", Langages, vol. 3, n¹1, 1968. p. 61-83; Jean de Bonville, Les quotidiens montréalais de 1945 à 1985 : morphologie et contenu, Québec, Institut québécois de recherche sur la culture, 1995.

3. L'objectif était d'obtenir un portrait plus précis que celui souvent fort général contenu dans l'ouvrage de référence usuel qu'est le «Beaulieu-Hamelin ». André Beaulieu et Jean Hamelin, La presse québécoise des origines à nos jours (tome 1 (1764-1859), tome 2 (1860-1879), tome 3 (1880-1895), tome 4 (1896-1910), tome 5 (1911-1919), tome 6 (1920-1934), tome 7 (1935-1940), tome 8 (1945-1954), tome 9 (1955-1963), tome 10 (1964-1975)), Québec, Presse de l'Université Laval, 1973-1990.). Ces résultats sont en cours de publication.

4. Ariane Santerre et Ève Léger-Bélanger, «L'image de l'Allemagne dans La Patrie et La Presse de l'entre-deux-guerres ", rapport de recherche.

5. Charles-Philippe Courtois, «Le séparatisme québécois des années 1930 et le nonconformisme ", Bulletin d'histoire politique, vol. 16, $n^{\circ} 2,2008$, p. 287-302.

6. Elmer W. Ferguson, « The Gist and Jest of It ", Montreal Herald, 4 août 1936.

7. Howard F. Stidwill, The History of the Canadian Olympic Association, mémoire de M.A (éducation physique), University of Ottawa, 1981.

8. Ibid., p. 72

9. «Histoire de l'athlétisme au Québec ", Fédération québécoise d'athlétisme [En ligne], < http:// athletisme-quebec.ca/histoire-de-lathletisme-au-quebec.php > (19 décembre 2014).

10. Rappelons que Montréal était une ville majoritairement anglophone de jusqu'en 1865 . «Montréal », Canadian Encyclopeadia, http://www.thecanadianencyclopedia.ca/en/article/ montreal/> Consulté le 9 janvier 2015. En 1931, la proportion des francophones y est d'environ 63\% (Paul-André Linteau, René Durocher, Jean-Claude Robert, François Ricard, Histoire du Québec contemporain. Le Québec depuis 1930, Montréal, Boréal, p.58.

11. Le Montreal Amateur Athletic Association est le club d'athlétisme le plus important au Québec pendant la première moitié du XXe siècle. Les autres clubs fondés dans les années 1930 ont aussi des noms en anglais (North End Eagles A.A., Québec Swimming and Athletic Club, Royal Rovers Harriers, etc.). Les liens sont étroits ente l'élite anglophone et le sport olympique. Ainsi, la Province of Quebec Track and Field Association, fondée en 1934, est dirigée par le directeur des sports de l'Université McGill, Arthur S. Lamb, jusqu'aux années 1950. Le deuxième club en terme d'importance historique est le Montreal Police A.A.A.

12. C'est ce que révèle une analyse systématique de la facture des journaux que nous avons effectuée au préalable, selon la méthode de la semaine recomposée. Nous avons étudié les années suivantes : 1919-1920, 1923-1924, 1928-1929, 1933-1934, 1938-1939. La publication des résultats ces analyses est prévue en 2017, sous la direction de Dominique Marquis et Micheline Cambron.

13. La réduction dramatique de l'accès aux archives du Musée de l'Amérique française n'a permis qu'un survol des numéros. Nous en avons tout de même tiré quelques informations pertinentes, comme on le verra. Nos remerciements vont à Ariane Santerre.

14. Les informations sur les tirages proviennent du Canadian Newspaper Directory de 1936 (A. McKim, McKim's directory of Canadian publications [microfilm], Canadian Institute for Historical Reproduction, 1936). Celles sur les orientations des journaux sont tirées de nos propres analyses, qui complètent les notices de l'ouvrage de Beaulieu et Hamelin La presse québécoise des origines à nos jours, op. cit.

15. Les journaux anglophones sont diffusés à Montréal, au Québec et dans le reste du Canada anglais, tandis que les journaux francophones sont diffusés au Québec, dans les communautés francophones du reste du Canada et aussi aux Etats-Unis, auprès de la communauté francoaméricaine. Le détail de la circulation extra-montréalaise est peu documenté.

16. Désigné comme le Star dans la suite du texte. Quotidien anglophone d'orientation conservatrice, tirage : 118 996. exemplaires par jour.

17. Quotidien francophone libéral à vocation populaire, propriété de La Presse, tirage : 18923 exemplaires en semaine et 39519 le samedi. 
18. Quotidien francophone résolument libéral, tirage : 14024 exemplaires par jour.

19. Quotidien francophone, associé au parti de l'Union nationale qui se spécialise dans la nouvelle locale illustrée et la nouvelle sportive, tirage : 16403 exemplaires.

20. Parfois orthographié Keneder Adler. Ce journal, de mouvance libérale, est publié en yiddish. Tirage : 19649 exemplaires par jour. Nous nous rallions à Pierre Anctil quant à l'orthographe Keneder Odler. Pierre Anctil, Through the Eyes of the Eagle. The Early Montreal Yiddish Press 1907-1916, Montréal, Véhicule Press, 2001, 208 p (édition et annotation de textes traduits du yiddish à l'anglais par David Rome).

21. Magazine mensuel francophone destiné à la famille, avec un tirage de 25627 copies par mois.

22. Nous avons traité succinctement et de manière globale les petites annonces et les cartes d'affaires.

23. Les photos-vignettes sont des images non intégrées à des articles, généralement accompagnées d'un texte explicatif ou un titre, contrairement aux photos incluses dans un texte.

24. Journal francophone d'information et de grande diffusion ayant de loin le plus important tirage : 144269 exemplaires en semaine, sans compter l'édition américaine.

25. Hebdomadaire francophone libéral radical, opposé au Parti Libéral et au Parti de l'Union Nationale. Volontiers anticatholique et antipatriotique. Pas de tirage connu.

26. Quotidien francophone, nationaliste et catholique, mais opposé à l'Union Nationale. Tirage : 14024 exemplaires.

27. Désigné comme la Gazette dans la suite du texte. Quotidien conservateur anglophone tiré à 28210 exemplaires.

28. Désigné comme le Herald dans la suite du texte. Quotidien anglophone au passé conservateur, il est plutôt libéral en 1936, mais il n'est tiré qu'à 19280 exemplaires.

29. Hebdomadaire tabloïd destiné à un public populaire, abondamment illustré et misant principalement sur les faits divers et les grands titres. Le journal appuie, à l'origine, le parti conservateur, mais il couvre largement le sport, la mode, le cinéma et, dans une moindre mesure, la vie culturelle. Tirage : 66343 exemplaires en septembre 1935.

30. Journal rédigé en italien, en français et en anglais, qui s'affiche ouvertement comme fasciste et dont la collection est fortement lacunaire. Il n'a pu être dépouillé comme prévu à cause de la réduction catastrophique des heures d'accès au Centre d'Archives du Musée de l'Amérique française à Québec.

31. Jean de Bonville, Les quotidiens montréalais de 1945 à 1985, op. cit.. p. 22-23, 198-203.

32. Nous avons distingué la photo-action, dans laquelle les athlètes sont représentés durant une épreuve ou lors de la remise des médailles, en pied ou en cadrage large, et les photos portraits, qui montrent la figure d'un athlète, dans un contexte qui n'est pas celui d'une performance.

33. Ils sont sans doute davantage un événement radiophonique. Il y a diffusion de la Cérémonie d'ouverture ( La plus formidable émission radiophonique du monde. Trois cents millions d'auditeurs pour les Jeux Olympiques. Détails de l'organisation fantastique ", Le Petit Journal, 9 août 1936, p. 46). et vraisemblablement d'autres formes de diffusion radiophonique auxquelles Georges Galipeau, chroniqueur radio à La Patrie fait écho. Un autre texte évoque l'importance des déploiements techniques liées à la radiophonie en Allemagne : Léo-Pol Morin, « La Rundfunkhaus de Berlin », Le Canada, 17 août 1936, p. 6. Nous n'avons pas exploré plus avant cette piste.

34. Dans le Star, 27 chroniques sur les 59 recensées portent sur les Jeux. Dans La Patrie, nos statistiques révèlent que près de $8 \%$ des éléments portant sur les Jeux sont des chroniques.

35. Lors des analyses préalables de la facture des journaux, nous n'avons trouvé aucun reportage. (cf. supra, note 12)

36. Ces chiffres reposent sur le nombre d'occurrences d'un objet à travers le corpus. Un même élément peut être associé à plus d'un objet.

37. Dans La Patrie, 80,8 \% des éléments sur les Jeux ont comme seul objet associé le sport. 
38. Exceptionnellement, certains articles proviennent d'autres villes comme Ottawa, Toronto, Londres ou Paris.

39. Désignée, selon les cas, Presse canadienne (P.C.), Canadian Press (C.P.) ou Canadian Press Cable (C.P.C.).

40. Associated Press (P.A. ou A.P.)

41. Désignée comme la U.P.

42. Désignée comme JTA.Cette agence diffuse des informations en anglais, lesquelles sont traduites par le journal en yiddish. Source : interview de M. Ira Robinson, janvier 2015. Rappelons que Montréal constitue l'une des villes américaines les plus importantes pour l'accueil d'immigrants juifs et que la vitalité culturelle et économique de cette communauté ethnique est aussi reconnue que celles de New York et Buenos Aires. Chantal Ringuet, À la découverte du Montréal Yiddish, Fides, 2011, p. 52-55.

43. Dans le cas du Canada, presque tous les éléments de notre corpus sont des tableaux de résultats pour lesquels la source n'est jamais indiquée. Puisque les nouvelles rendant compte de manière détaillée des Jeux ont pour source la Presse canadienne, on peut supposer que ces éléments proviennent de la même agence de presse.

44. Aucune signature n'apparaît dans Le Devoir, sauf celle de Lisa Gress pour son article intitulé «Le rôle de la femme dans les Jeux Olympiques », Le Devoir, $1^{\mathrm{er}}$ août 1936, p. 11

45. David Halton, Dispatches from the front: the life of Matthew Halton, Canada's voice at war, McClelland \& Stewart, 2014. Nous avons pu contre-vérifier cette information à partir de la liste des journalistes accrédités trouvée au CIO par nos collègues suisses. Qu'ils soient ici remerciés.

46. Plusieurs des correspondants qui signent des articles depuis Berlin dépendent d'agences de presse. Henry McLemore, publié dans le Star, et Elmer Dulmage, rédacteur sportif qui publie ses articles simultanément dans La Presse, la Gazette et le Star, sont mandatés respectivement par la United Press et la Presse canadienne. Des articles de Louis-P. Lochner de la Presse Associée et Stuart Cameron de la United Press paraissent dans La Presse.

47. Le Herald, 7 août 1936; « Secret Police Trail Olympic Writers » (U.P.), le Star, 7 août 1936, p. 17; "La police berlinoise surveille de près les journalistes ", Le Canada, 8 août 1936, p. 8; « Les potins de Berlin », La Patrie, 7 août 1936, p. 20; Tous ces textes reprennent un article originellement publié dans le London Daily Herald, le 6 août 1936 (non vidi).

48. «La police berlinoise surveille de près les journalistes ", Le Canada, loc. cit. On pourrait voir là un effet de la propagande anti-nazie britannique. Mais les travaux récents sur les Jeux de Berlin confirment le contrôle du régime nazi, en particulier en ce qui a trait aux images des JO. Voir à ce propos les autres articles du dossier.

49. Jean-Marie Brohm, 1936 - Jeux Olympiques à Berlin, Bruxelles, André Versaille éditeur, 2008, p. 12.

50. « Brève courtoisie envers les Juifs en Allemagne », L'Autorité, 8 août 1936, p. 4.

51. «Elle aimait trop le bar », La Patrie, 8 août 1936, p. 51.

52. P.A., "Disqualifié par l'Union Ath. Amateur, Jesse Owens deviendrait professionnel ", Le Canada, 17 août 1936, p. 11.

53. « [Si les américains n'avaient pas leurs athlètes noirs] », La Patrie, 6 août 1936, p. 21.

54. Câble Presse Canadienne, "Trois pays ont failli faire cause commune avec le Pérou ", La Patrie , 12 août 1936, p. 20.

55. « Helen Stephens est bien une femme ", L'Illustration nouvelle, 7 août 1936, p. 22.

56. Une foule immense assiste à la clôture des $\mathrm{XI}^{\mathrm{e}}$ Jeux Olympiques, hier à Berlin », Le Canada, 17 août 1936, p. 11.

57. « MM. Cattarinich et Dandurand dans le ministère sportif? », La Patrie, 16 août 1936, p.79.

58. Voir « Rapports de pouvoir : race, genre et nation dans la couverture montréalaise des JO de Berlin », dans ce numéro. 
59. Jean- François Nadeau affirme que, à partir d'octobre 36, le Fasciste canadien qu'il dirige est produit à partir des presses de L'Illustration nouvelle, et que « les idées fascistes [d'Arcand] sont alors connues, mais qu'il n'en fait pas étalage ». Adrien Arcand, führer canadien, Montréal, Lux éditeur, 2010, p. 142. Jean-François Nadeau nous a confirmé qu'Arcand est le rédacteur en chef du journal durant les Jeux Dans sa thèse, Mathieu Noël le confirme, soulignant que ce statut n'est toutefois pas officialisé («Le Montréal-Matin (1930-1978). Un journal d'information populaire », thèse de doctorat (PH.D.) en histoire, UQAM, 2014, p.85). Noël donne aussi de précieuses informations, tirées de diverses correspondances, dont celle-ci: «le propriétaire, Eugène Berthiaume, a fait en sorte que son journal prenne discrètement un abonnement à un service de presse nazi » (op.cit., p. 91).

60. Presse Canadienne, «Une foule immense assiste à la clôture des XIe Jeux olympiques, hier à Berlin », Le Canada, 17 août 1936, p. 11; "L'Allemagne remporte les honneurs aux olympiques », L'Illustration Nouvelle, 17 août 1936, p. 21.

61. «Un troisième triomphe pour Jesse Owens ", L'Illustration nouvelle, 6 août 1936, p. 21.

62. "Owens is Triple Olympic Winner », The Montreal Daily Star, 5 août 1936, p. 21.

63. «Jesse Owens reste la vedette des Jeux Olympiques ", La Patrie, 6 août 1936, p. 20.

64. "Jesse Owens fait sensation aux Jeux - Phil Edwards se classe 3ième dans les 1,500 et 800 mètres - Lee Orr est 5ième dans le sprint », 6 août 1936, p. 11.

65. Arcand déclare dans une lettre à Berthiaume éviter de soutenir explicitement l'Union nationale dans les éditoriaux mais la favoriser " dans les nouvelles et les rapports » (Mathieu Noël, op. cit. p. 95). Il use en ce cas du même type de stratégie, manipulant la nouvelle.

66. «Canadians Shine in Olympic - Jesse Owens Wins 200-Metre Final - Orr Flashes form Reaches 200 Final ", The Montreal Daily Star, 5 août 1936, p. 19.

67. « Jesse Owens fait sensation aux Jeux », Le Canada, 6 août 1936, p. 11.

68. "Les Japonais dominent toujours dans la nage ", L'Illustration nouvelle, 12 août 1936, p. 22. Notons que cette expression est reprise telle quelle dans le numéro du Canada de la même journée. Il semble que les comptes rendus que publient ces deux journaux soient à peine démarqués du même article en anglais.

69. « Les nègres remportent une victoire complète à Berlin ", L'Illustration nouvelle, 8 août, p. 21.

70. « Réflexions brèves ", La Patrie, 5 août 1936, p. 8.

71. "Potins de Berlin », La Patrie, 6 août 1936, p. 20

72. « Don précieux aux Allemands », La Presse, 5 août 1936, p. 16.

73. «Canadian Shines in Half-mile Final », The Montreal Daily Star, 4 août 1936, p. 19; « 2 records mondiaux tombent à l'Olympiade de Berlin », La Patrie, 3 août 1936, p. 20; " Edwards et Loaring gagnent des points pour le Canada aux Jeux Olympiques ", Le Canada, 5 août 1936, p. 11; "La fameuse constellation de la piste américaine remporte, hier, son 3e titre dans le 300 mètres », La Patrie, 6 août 1936, p. 20.

74. «Le Japon gagne la majorité des courses de nage », L'Illustration nouvelle, 10 août 1936, p. 21; «Frank Amyot, d'Ottawa, gagne le simple du canotage», La Patrie, 10 août 1936, p. 20; «L'Allemagne remporte les honneurs aux Olympiques », L'Illustration nouvelle, 17 août 1936, p. 21. 75. « Nos espérances pour aujourd'hui », La Patrie, 11 août, p. 20.

76. « Nos inscrits aujourd'hui », La Patrie, 7 août, p. 20.

77. Horace Lavigne, « Au panthéon des sports - Nos gloires, leurs exploits », 9 août 1936, p. 68-69.

78. « What Canada Did at Olympic Today », Montreal Daily Star, 14 août 1936, p. 20.

79. « How Canadian Fared Yesterday », Montreal Daily Star, 15 août 1936, p. 8.

80. «Les chances du Canada aujourd'hui », Le Canada, 4 août 1936, p. 11.

81. Henry McLemore, "Novel Events Boost Points for Germany ", Montreal Daily Star, 15 août 1936, p. 8.

82. Beaucoup d'autres textes de La Revue moderne sont écrits au «nous ", celui de la collectivité « canadienne-française ». 
83. Georges Laferrière, «Le Brasseur futur poids-lourd », La Revue moderne, janvier 1936, p. 25.

84. Henry McLemore, « Weird Words Used to Cover Baseball », Montreal Daily Star, 14 août 1936, p.

20.

85. Henry McLemore, « Weight Man Wear Santy Mat Togs », Montreal Daily Star, 6 août 1936, p. 25.

86. Henry McLemore, « Only Miracle Can Keep U.S. From Top », Montreal Daily Star, 8 août 1936, p. 8.

87. «Le héros de la première victoire canadienne aux Jeux Olympiques », Le Petit Journal, 16 août 1936, p. 56. Il est précisé "Spécial au "Petit Journal” ». Cette mention revient pour deux autres articles : «Les Jeux Olympiques de Berlin sont commencés », Le Petit Journal, 2 août 1936, p. 50; Le vélodrome olympique est terminé ", Le Petit Journal, 2 août 1936, p. 50.

88. L. Berek, « 'Dos Yidishe Leben in Frankraych' - La vie juive en France », Keneder Odler, 14 août 1936, p. 4, 8.

89. Voir Marie-Ève Thérenty, "La chronique ou l'écho dérouté », 1836. L'An I de l'ère médiatique, Paris, Nouveau monde éditions, 2001, p. [264]-276.

90. Georges Galipeau, « À l'écoute à CHLP », La Patrie, 4 et 6 août 1936, p. 15.

91. Paul Parizeau, «Réflexes et commentaires », Le Canada, 12 août 1936, p. 11.

92. Myrtle Cook, The Montreal Daily Star, «In the Women's Sportlight », 7 août 1936, p. 18; Baz O'Meara, « The Passing Sport Show », Montreal Daily Star, 8 août 1936, p. 10.

93. Dunc MacDonald, « Sport On Parade », The Gazette, 1er août 1936, p. 13.

94. Zotique Lespérance, « Nouvelles et commentaires sportifs », La Patrie,16 août 1936, p.76.

95. Zotique Lespérance, « Nouvelles et commentaires sportifs », La Patrie, 13 août, p. 21

96. Scaramouche, «Eh non, les purs aryens ne sont pas des surhommes! », L'Autorité, 22 août 1936, p. 1. L'Autorité n'utilise que des pseudonymes comme signatures.

97. A.S. tient une chronique hebdomadaire, «Mit Zalts un Fefer» Sur les deux chroniques de notre corpus, une seule parle des JO.

98. A.S. « Mit Zalts un Fefer ", Kender Odler, 13 août 1936, p. 4.

99. Selon un spécialiste de la caricature, Dominic Hardy, Herblock est un caricaturiste américain lié au Newspaper Enterprise Association, très présent dans le Herald des années trente.

100. Herblock, The Torch bearers, The Montreal Herald, 10 août 1936, p. 4.

101. «There off!", The Montreal Star, 3 août, 1936 p. 10 ; " Maskrad à Naziland », Keneder Odler, 3 août, 1936, p. 4.

102. Nous avons relevé, au cours de la période des Jeux, 55 occurrences du mot « nazi » ou de ses dérivés dans le Keneder Odler, par exemple, " 'Natsis Velen Aroysgeben Aliles-Dam Buch Noch Olimpiade' - Les nazis veulent distribuer un livre d'accusation de sang après l'Olympiade ", Keneder Odler, 2 août 1938, p. 8; " 'Tsulib di Olimpiks iz Men in Daytshland "Heflich" tsu Yiden' - À cause des Olympiques on est "poli" en Allemagne avec les juifs ", Keneder Odler, 5 août 1936, p. 4. «'Natsi Gerichten Hoben Shoyn Far-Mishpet 225 Toyzent Man' - Les cours nazies ont déjà jugé 225 milles personnes ", Keneder Odler, 6 août 1936, p. 1.

103. « Maskrad in Natsi Land» (« Mascarade dans la Terre des nazis »), Keneder Odler, 3 août 1936, p. 4.

104. « Jeux olympiques ", La Presse, 4 août 1936, p. 6.

105. «There off !", The Montreal Star, 3 août, 1936 p. 10.

106. La Presse, loc.cit., p. 6.

107. Ibid., p. 6.

108. Zotique Lespérance, « Nouvelles et commentaires sportifs » La Patrie, 13 août, p. 21

109. Ibid.

110. Fabrice d'Almeida et Christian Delporte, « « Médias, propagande et patriotisme », Histoire des médias en France, Paris, Flammarion, p. 37-41.

111. Paul Parizeau, « Réflexes et commentaires », 12 août, p.11. 
112. La crise entraîne, entre 1933 et 1935 , des disparitions de journaux, des changements de propriétaire, et, globalement, un appauvrissement de l'information journalistique.

\section{ABSTRACTS}

Les Jeux olympiques de Berlin en 1936 sont l'un des moments forts de la lutte idéologique qui se déroule alors sur la scène internationale et Montréal n'y échappe pas. Le présent article explore l'hypothèse que cet événement aurait rendu plus lisibles les clivages identitaires et politiques de la société québécoise, à partir de l'analyse de la couverture qu'offre des Jo un échantillon substantiel de journaux montréalais. La première partie de cette étude décrit les caractéristiques de la couverture des Jeux, replacée sur la facture usuelle des journaux, et révèle la dépendance des périodiques montréalais à l'endroit des agences de presse nord-américaines. Le recours massif aux agences crée l'illusion d'un récit unique ressassé partout, fait de tableaux et de petites anecdotes. Cependant, grâce aux textes dans lesquels s'affirme une subjectivité, dans les chroniques sportives, entre autres, le lecteur de l'époque est mis en contact avec l'expression de jugements individuels desquels se dégage une interprétation proprement montréalaise des Jeux olympiques, qui fait une place à des enjeux débordant le caractère strictement sportif de l'événement pour toucher aux questions de politique internationale. L'hypothèse initiale se révèle finalement insatisfaisante, les JO paraissant avoir joué le rôle d'un catalyseur modifiant provisoirement l'économie du système des journaux montréalais, réorganisé autour d'impératifs moraux irréductibles aux clivages sociaux et identitaires locaux.

\section{INDEX}

Mots-clés: Jeux olympiques, Montréal, Québec, médias, journaux, sport

\section{AUTHORS}

\section{XAVIER BOILEAU}

Xavier Boileau est étudiant en maîtrise de philosophie à l'Université de Montréal. Publications : «Trahison et citoyenneté : L'utilisation des procès militaires pour redéfinir l'espace politique au Bas-Canada entre 1838 et 1840 », dans Revue Le Manuscrit édition colloque 2014 (http:// www.revuelemanuscrit.uqam.ca/index.php/edition-colloque/vi-colloque-2014/60-trahison-etcitoyennete-l-utilisation-des-proces-militaires-pour-redefinir-l-espace-politique-au-bas-canadaentre-1838-et-1840) ; « Dewey et Gutnam : l'éducation civique comme nécessité démocratique », dans Ithaque, n. 19, 2016, p. 3-24

\section{JEANNE BOUCHER LAUZON}

Jeanne Boucher Lauzon est étudiante à la maitrise en littératures de langue française à l'Université de Montréal. 


\section{MICHELINE CAMBRON}

Micheline Cambron est professeure au Département des littératures de langue française de l'Université de Montréal et membre du Centre de recherche interuniversitaire sur la littérature et la culture québécoises (CRILCQ). Elle consacre ses recherches et son enseignement à la littérature et à la culture québécoises des $\mathrm{XIX}^{\mathrm{e}}$ et $\mathrm{XX}^{\mathrm{e}}$ siècles. Elle a principalement travaillé sur les formes de l'utopie, les relations presse/littérature et les questions d'histoire littéraire et culturelle. Elle est également spécialiste des œuvres de Fernand Dumont et Paul Ricœur.

\section{DOMINIQUE MARQUIS}

Dominique Marquis est professeure d'histoire à l'Université du Québec à Montréal. Spécialiste d'histoire de la presse québécoise et d'histoire socioreligieuse du Québec, elle a publié plusieurs articles sur la presse au Québec Elle a participé au projet Histoire du livre et de l'imprimé au Canada / History of the Book in Canada. Elle est l'auteure de Un quotidien pour l'Église : L'Action catholique, 1910-1940, paru en 2004 aux Éditions Leméac. Elle mène actuellement un projet de recherche sur Jules-Paul Tardivel et La Vérité, un réseau ultramontain au service d'une œuvre. Elle est membre du groupe de recherche " La presse montréalaise et la vie culturelle dans l'entre-deux-guerres » et du Groupe de recherches et d'études sur le livre au Québec (GRELQ).

\section{ALEX GIROUX}

Alex Giroux est étudiant à la maitrise en histoire à l'Université du Québec à Montréal.

\section{EVE LÉGER-BÉLANGER}

Eve Léger-Bélanger est étudiante au doctorat en littératures de langue française à l'Université de Montréal. Publications : «Le corps de la jeune fille au début du XXe siècle : une différenciation marquée selon les auteurs masculins ou féminins ? ", Les Savoirs des femmes [en ligne], avril 2015. URL : http://savoirdesfemmes.org/public_html/?page_id=161; « La théâtralisation du pâtir dans la pièce de L'Ile de la demoiselle d'Anne Hébert ", Actes de colloque Les XXVIIes Journées de la Linguistique [en ligne], Université Laval, mars 2013. URL : http://jdl.lli.ulaval.ca/documents/ actes/actes_2013_Leger-Belanger.pdf ; «Quand conquête terrestre devient conquête sur papier : l'Allemagne dans La Presse en mai 1940 ", Micheline Cambron, Myriam Côté et Alex Gagnon (dir.), Les journaux québécois d'une guerre à l'autre. Deux états de la vie culturelle québécoise au XXe siècle, Québec, Éditions Codicille (sous presse) ; «L’Allemagne dans La Presse et la littérature québécoise de l'entre-deux-guerres : une représentation univoque?», Actes du Colloque International Interdisciplinaire de l'Association des Etudiant-e-s Diplômé-e-s du Département d'histoire de l'Université de Montréal, Conflits et sociétés : des armes aux discours (mai 2014), Université de Montréal, Cambridge Scholars Publishing, directeur du dossier : Nari Shelekpayev (accepté pour la publication) ; « Paradis d'Abdulrazak Gurnah : une culture de l'échange », Actes du colloque international (E)migrations, transferts, exils :métissages et dynamiques de la ville (avril 2015), Université Masaryk de Brno (République Tchèque), Etudes romanes de Brno (soumis) ; «Fascination et catégorisation : construction de l'espace et du lieu liés à l'Allemagne dans la presse et la littérature québécoises de l'entre-deux-guerres ", Actes du colloque Parcourir, cartographier, habiter : les lieux en littérature - Colloque étudiant du Département des littératures de langue française de l'Université de Montréal (janvier 2015), Sens public [en ligne] (soumis). 


\section{JÉRÉMI PERRAULT}

Jérémi Perrault est étudiant au doctorat en littérature de langue française et à l'Université de Montréal. Publications : Dans la gueule du jour », Spirale, n² 253, été 2015, p. 63-64 ; « La fin du sentier ", Spirale, $n^{\circ} 250$, automne 2014, p. 41-42.

\section{YOSEF ROBINSON}

Yosef Robinson est doctorant en études judaïques à l'Université d'Ottawa. Publications : Compte rendu de livre, Social Theory - A Historical Analysis of Canadian Socio-cultural Policies, 'Race' and the 'Other', Chales Asher Small, été 2014. A publier dans Etudes juives canadiennes, 2015 ; Ecumenism, «An Organizational Flowering ", publié par le Centre canadien d'œcuménisme, Montréal, automne 2011, \#183, coécrit avec Ira Robinson. Version modifiée de l'article du 15 août 2010, n 69 ; Changing Jewish Communities, « Environmental Activism in the Canadian Jewish Community ", publié par l'Institute for Global Jewish Affairs, Jérusalem, 15 août 2010, \#59, coécrit avec Ira Robinson ; Dateline : Middle Est (magazine), « The Israeli-Palestinian Situation at Rutgers », publié par The Student Coalition for a Just Peace in the Middle East, Montréal, printemps 2004, volume 19, page 5.

\section{MARILOU ST-PIERRE}

Marilou St-Pierre est candidate au doctorat en communication à l'Université Concordia. Publications : Charron Jean, Marilou St-Pierre et Geneviève Drolet. 2015. « La parole des femmes dans les journaux télévisés au Québec, 1961-2010 ", Communication [En ligne], vol. 33/1 | 2015, mis en ligne le 16 février 2015. URL : <http://communication.revues.org/5127> ; DOI: 10.4000/ communication.5127; « Women Sports Reporters : Feminity in a traditional male field ». Dans II International Conference Gender and Communication, Conference proceeding, 2014, p. 50-63 ; Brin, Colette et Marilou St-Pierre. 2013. "Crise des médias et effectifs rédactionnels au Québec », Centre d'études sur les médias, $60 \mathrm{p}$. 